\title{
PRE-AMP BOX PLATFORM ANALYSIS
}

D-Zero Engineering Note: 3740.210-EN-198

K. Kirby

C. Kurita

January 17, 1989

Approved: 20 Sewn $1 / 17 / 89$ 


\section{INTRODUCTION}

A platform to be used for the installation and repair of the high voltage pre-amp boxes on the $\mathrm{CC}$ cryostat has been designed to support a uniform load of $30 \mathrm{lbs} . / \mathrm{sq}$. ft. However, according to the standards set by both the American National Standard and the Uniform Building Code, the minimum uniformly distributed design load for a structure used as an "elevated platform or walkway" is 60 lbs./sq. ft. The existing platform was tested with a uniform load of $40 \mathrm{lbs} . / \mathrm{sq}$. ft. with no major problems occurring during the testing. Considering a $40 \mathrm{lbs} . / \mathrm{sq}$. ft. load to be the minimum acceptable value for "residential" use, and the platform in hand to be better categorized as an "elevated platform or walkway", the platform is carefully re-analyzed for a $60 \mathrm{lbs} . / \mathrm{sq}$. ft. uniformly distributed load.

\section{PLATFORM MODELING}

The platform, which is made entirely of aluminum, measures $107 \mathrm{in.}$ by $36 \mathrm{in.}$ It is constructed of a frame of $1-1 / 2 \mathrm{in.}$ by $3 / 4 \mathrm{in}$. by $1 / 8$ in. (wall) rectangular tubing with flats welded to the its top and bottom. The top plate is $1 / 8$ in. thick and the bottom plate is $1 / 16$ in. thick. The following drawings show the detail of the platform and its support assembly:

$3740.213-\mathrm{ME}-273362$

$3740.213-\mathrm{ME}-273363$

$3740.213-\mathrm{ME}-273364$
D-0 PRE-AMP BOX PLATFORM AND SUPPORT ASSEMBLY

D-0 DETECTOR PRE-AMP BOX PLATFORM WELDED UNIT

D-0 DETECTOR PRE-AMP BOX SUPPORT FRAME WELDED UNIT 
A finite element model has been designed using ANSYS to simulate the platform deflections and material stresses under the application of a $60 \mathrm{lbs} . / \mathrm{sq}$. ft. uniform load. The model consists of two element types. The first is quadrilateral shell elements (stif 63), which were used for the plates, and the second is 3-d beam line elements (stif 4), which were used for the frame. An effective inertia was determined and entered into the program to account for shear lag. See Appendix A for inertia calculations. Since the support pins are welded to the gussets and go through both the bottom and top plate, it effectively gives us this displacement boundary condition at the supports. The nodes which are located where the gussets will be lying are restrained in the negative y-direction only. The holes present in the plates at the locations of the support pins do not pose a stress concentration problem when loaded because they are not located in the area where the beam frame is and therefore do not effectively take away from the cross-sectional area used in the analysis.

The platform was also modeled using $B E A M A C$, with the crosssection of the beam consisting of the rectangular tubing attached to the top and bottom plates times four. A uniform distributed load of $15 \mathrm{lbs} . / \mathrm{in}$.(as determined from the application of a $60 \mathrm{lbs} . / \mathrm{sq}$. ft. load applied to the area of the platform, divided by the length of the beam) was applied to the beam. The same effective inertia used in the ANSYS model was used for the beam elements.

\section{ANSYS AND BEAMAC RESULTS}

The model used in the ANSYS analysis and the appropriate maximum displacements and stresses can be found in Appendix B, and the input and output data used in the $B E A M A C$ model is located in Appendix C.

After loading the model, the maximum deflection in the platform, as determined by ANSYS, was .4355 in., occurring at the center of the platform (node 289). The maximum deflection experienced by the beams near the center node of the structure was 
.4203 in. This value should be compared which the results from $B E A M A C$. The maximum bending stress in the frame was 5156.3 psi. This was located in the horizontal beams located near the center of the structure (element 497/nodes $229 \& 230$ ). Since the maximum allowable stress is equal to sixty percent of the yield strength (40 ksi for aluminum), which is $24 \mathrm{ksi}$, the structure has an adequate factor of safety (4.65).

Upon cooling, some of the plug welds in the bottom plate failed. As a result, skip welds were added along the perimeter of the bottom plate. However, if these welds are viewed as being failed, essentially excluding the stiffness of the bottom plate, there will be a $23 \%$ increase in deflection and $18 \%$ increase in the platform stress. Under this condition the structure still has an adequate safety factor of (3.77).

The maximum deflection of the model obtained from $B E A M A C$ was .3010 in., occurring in the center of the beam. From the maximum moment (4040 lb.-in.), the maximum bending stress was calculated to be 5297 psi, also located at the center of the beam. These values are comparable to those obtained from ANSYS. The difference in the displacement values can probably be accounted for by the fact that the maximum displacement in the ANSYS model does not occur where a cross-beam is located, but rather in the middle of the plate. The nodal plot and displacement listings show that the displacement values are slightly lower at the location of the beams.

\section{CONCLUSION}

Although the platform was originally designed for a load of 40 lbs./sq. ft., further analysis has demonstrated that the platform can safely handle a load of $60 \mathrm{lbs} . / \mathrm{sq}$. ft. and still maintain a sufficient factor of safety. To help insure that the platform is not misused and overloaded, a sign stating a maximum load value will be clearly posted. 


\section{APPENDIX A}

\section{Effective Moment of Inertia:}

This calculation will determine the effective moment of inertia for a composite constructed of a $1-1 / 2$ in. by $3 / 4$ in. by $1 / 8$ in. wall box beam, a $1 / 8$ in. top plate, and a $1 / 16$ in.bottom plate. We will be using an effective width of $10 \mathrm{t}$ on either side of the shear attachment to account for shear lag in the plates. This is a considered a conservative approach.

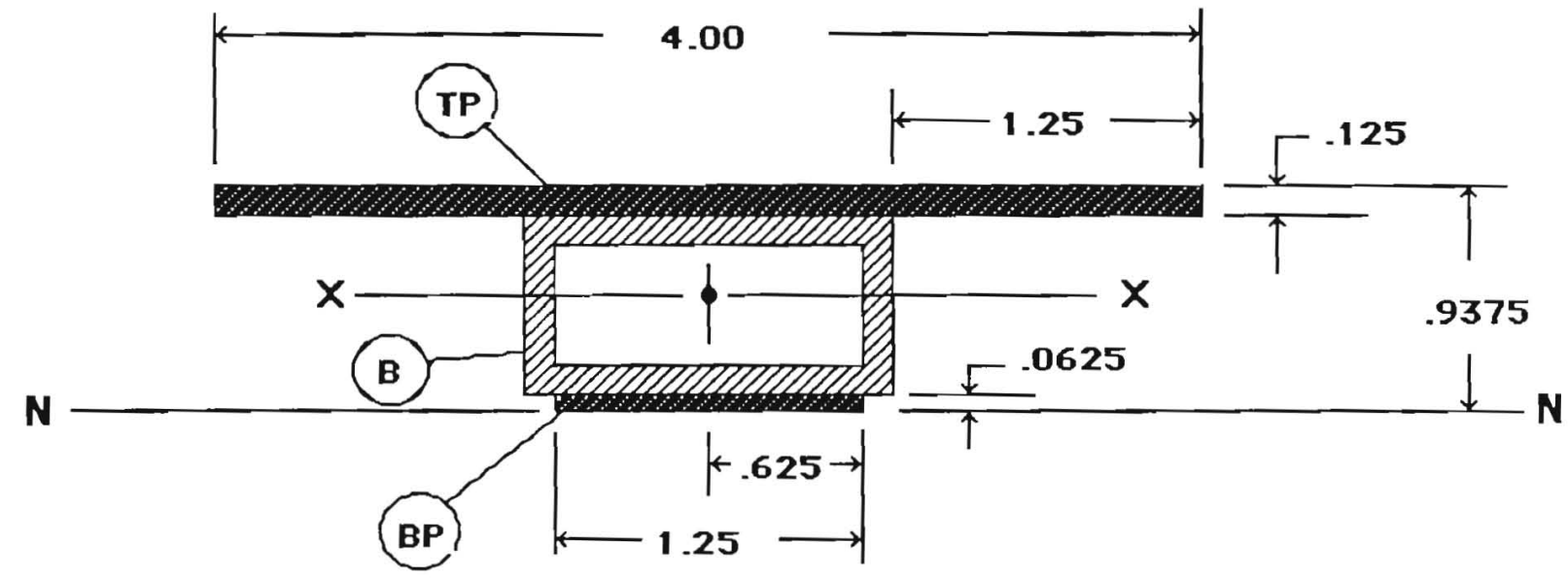

Ix (b) $=.03971$ in.^4 $\quad$ Iy (b) $=.1296$ in.^4

$\mathrm{A}(\mathrm{b})=.500$ in..$^{\wedge} 2$

Ix $(\mathrm{tp})=.000651$ in. ${ }^{\wedge} 4$

Iy $(\mathrm{tp})=.6667$ in.^4

$\mathrm{A}(\mathrm{tp})=.500 \mathrm{in} .^{\wedge} 2$

Ix $(b p)=.0000254$ in.^4

Iy $(\mathrm{bp})=.0102$ in.^4

$\mathrm{A}(\mathrm{bp})=.07813$ in..$^{\wedge}$

$\mathrm{y}(\mathrm{b}) \mathrm{n}=.4375 \mathrm{in}$.

$\mathrm{y}(\mathrm{tp}) \mathrm{n}=.875 \mathrm{in}$.

$\mathrm{y}(\mathrm{bp}) \mathrm{n}=.03125 \mathrm{in}$.

Effective Area (Aeff):

$$
\text { Aeff }=A(b)+A(t p)+A(b p)=1.07813 \text { in. }{ }^{\wedge} 2
$$

\section{Centroid of Composite ( $\left.Y_{c}\right)$ :}

$$
\mathrm{Yc}=\mathrm{A}(\mathrm{b}) \mathrm{y}(\mathrm{b})+\mathrm{A}(\mathrm{tp}) \mathrm{y}(\mathrm{tp})+\mathrm{A}(\mathrm{bp}) \mathrm{y}(\mathrm{bp}) / \mathrm{Aeff}=.6110 \mathrm{in} \text {. }
$$

\section{Moments of Inertia about Neutral Axis (In):}

$$
\text { In }(b)=\operatorname{Ix}(b)+A(b) y(b)^{\wedge} 2=.1354 \text { in.^4 }
$$




$$
\begin{aligned}
& \text { In }(t p)=\operatorname{Ix}(t p)+A(t p) y(t p)^{\wedge} 2=.3830 \text { in.^4 } \\
& \text { In }(b p)=I x(b p)+A(b p) y(b p)^{\wedge} 2=.0001017 \text { in. } .^{\wedge} \\
& \text { In (total) }=\text { In (b) }+\operatorname{In}(t p)+\operatorname{In}(b p)=.5190 \text { in. } .^{4}
\end{aligned}
$$

Effective Composite Moment of Inertia about the $\underline{\text { axax }}$ (Ieffx):

$$
\text { Ieff } x=\text { In (total) }+\operatorname{Aeff}(Y c)^{\wedge} 2=.1165 \text { in. } .^{\wedge}
$$

This value of inertia about the $\mathrm{x}$-axis will be used in our model.

Effective Composite Moment of Inertia about the v-axis (Ieffy):

$$
\text { Ieffy }=\text { Iy (b) }+ \text { Iy (tp) }+ \text { Iy (bp) }=.8065 \text { in.^4 }
$$

Effective Moment of Inertia (plug failure):

$$
\begin{aligned}
& \text { Ieff } x=.0882 \text { in.^4 } \\
& \text { Ieffy }=.7963 \text { in.^4 }
\end{aligned}
$$




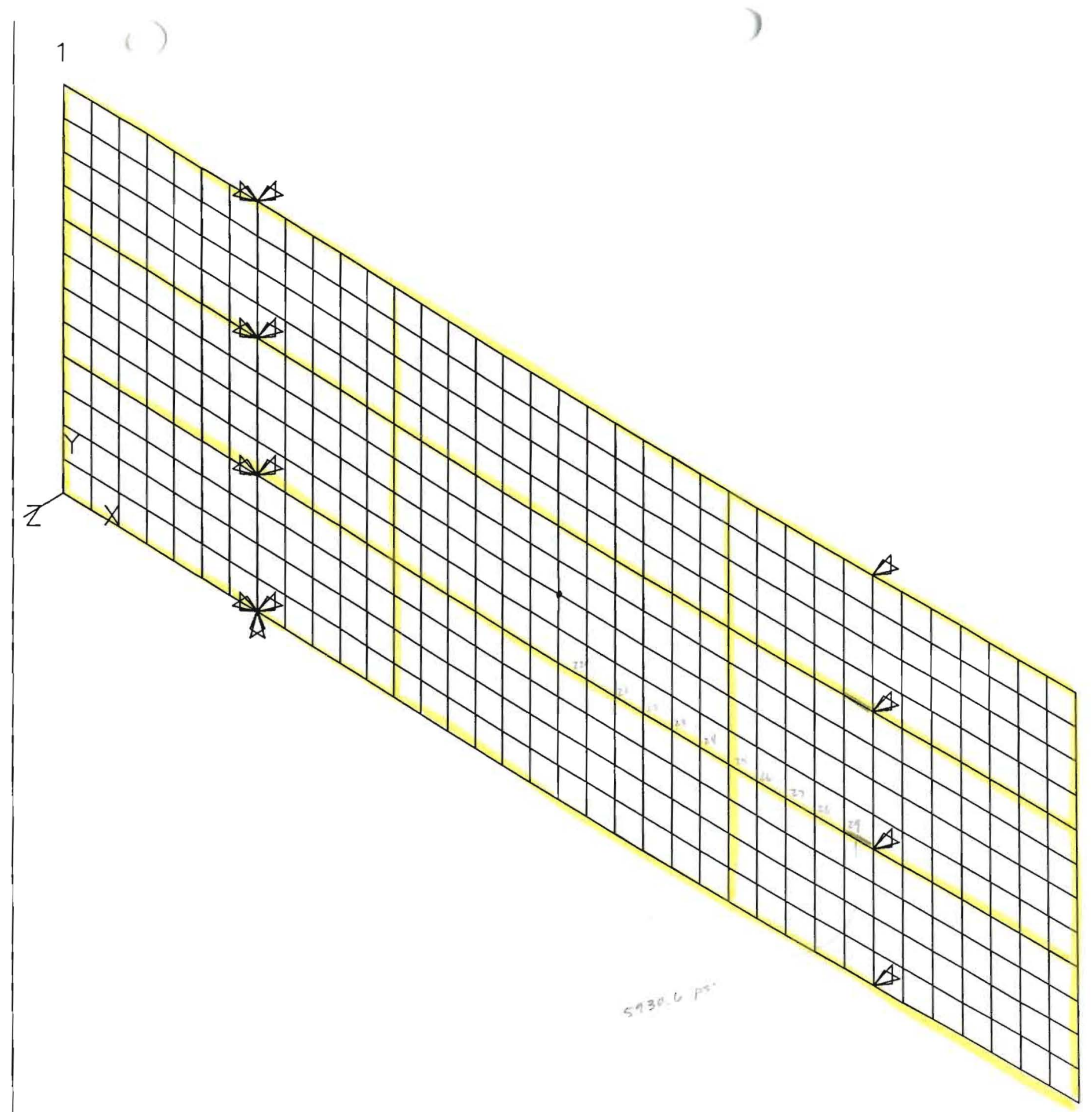

ANSYS 4.3A

DEC 281988

$\begin{array}{lll}14 & 09 & 44\end{array}$

PLOT NO. 1

PREP7 ELEMENTS

$$
\begin{aligned}
& X V=1 \\
& Y V=1 \\
& Z V=1
\end{aligned}
$$

$\mathrm{DIST}=42.003$

$X F=54$

$Y F=18$

DREAMS

$(1120 \times 3 / 4 \times 1 / 8 " w A L L)$

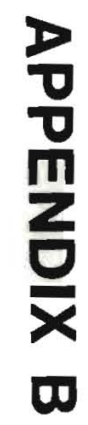




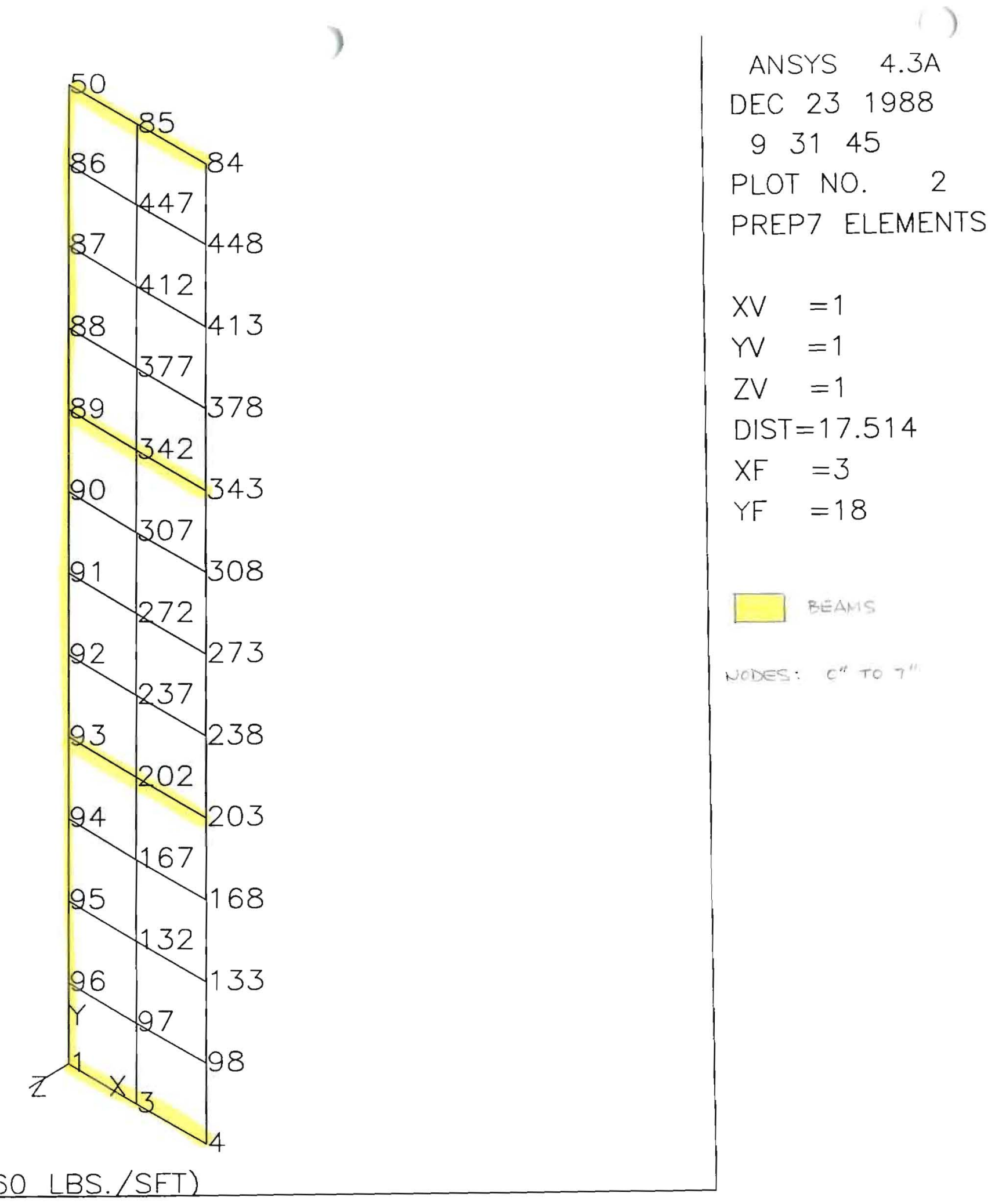




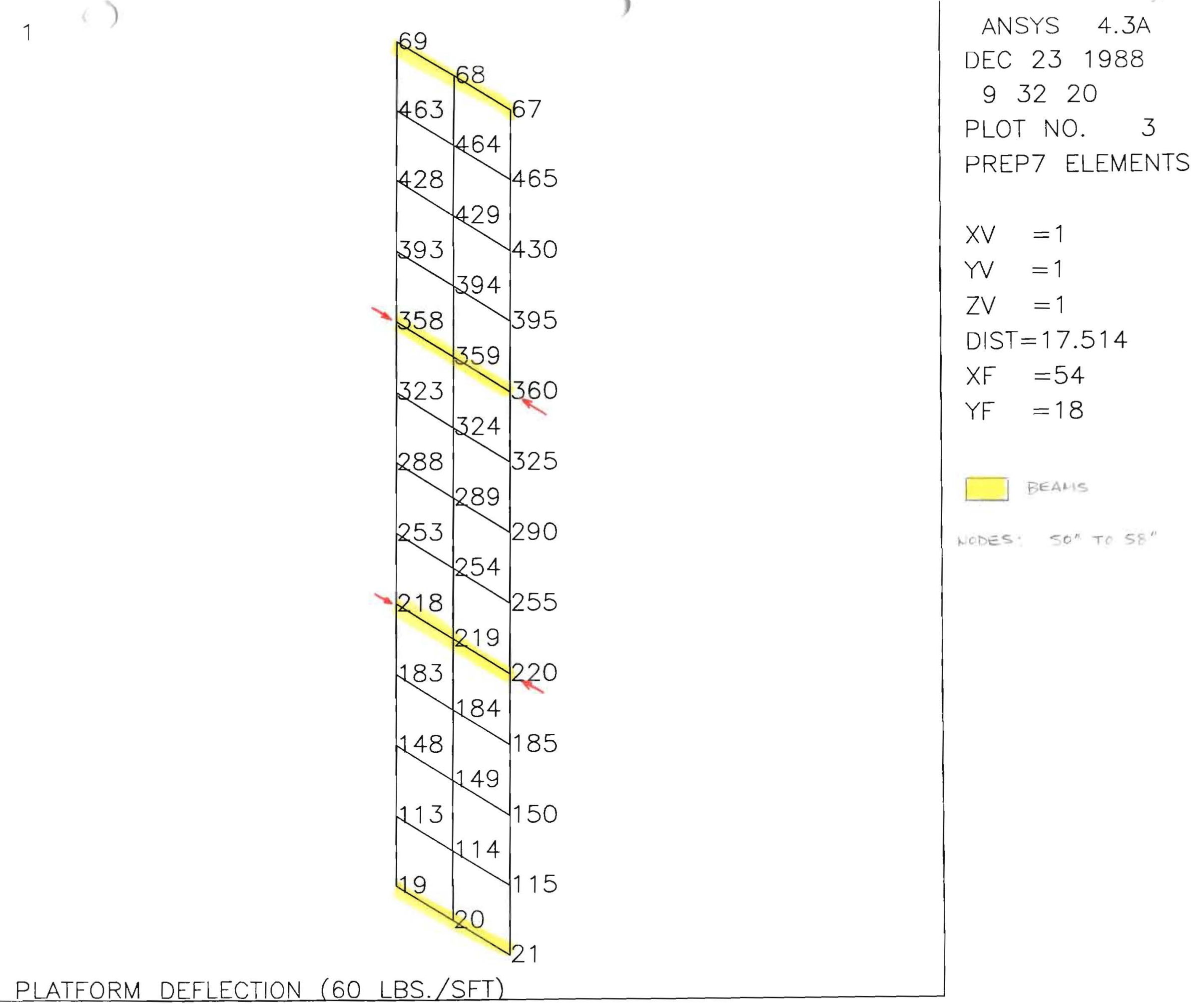




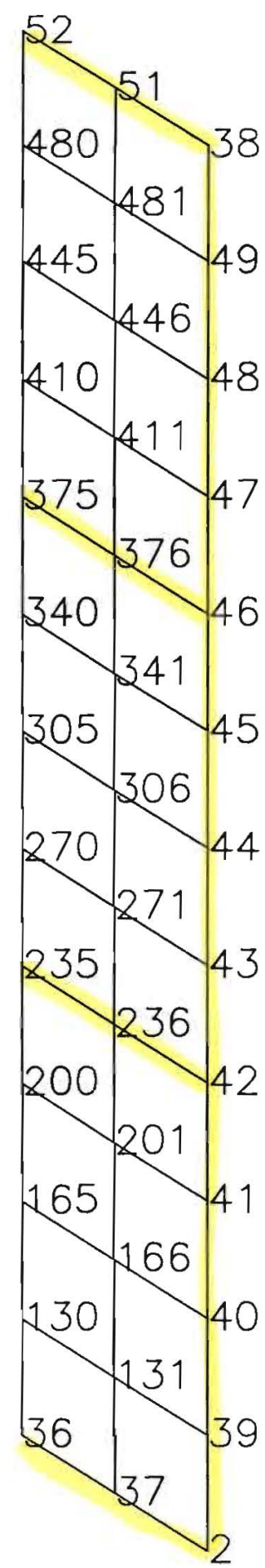

ANSYS 4.3A DEC $23 \quad 1988$

$\begin{array}{lll}9 & 32 & 48\end{array}$

PLOT NO. 4

PREP7 ELEMENTS

$X V=1$

$Y=1$

$\mathrm{ZV}=1$

$\mathrm{DIST}=17.514$

$X F=105$

$\mathrm{YF}=18$

PLATFORM DEFLECTION (60 LBS./SFT) 


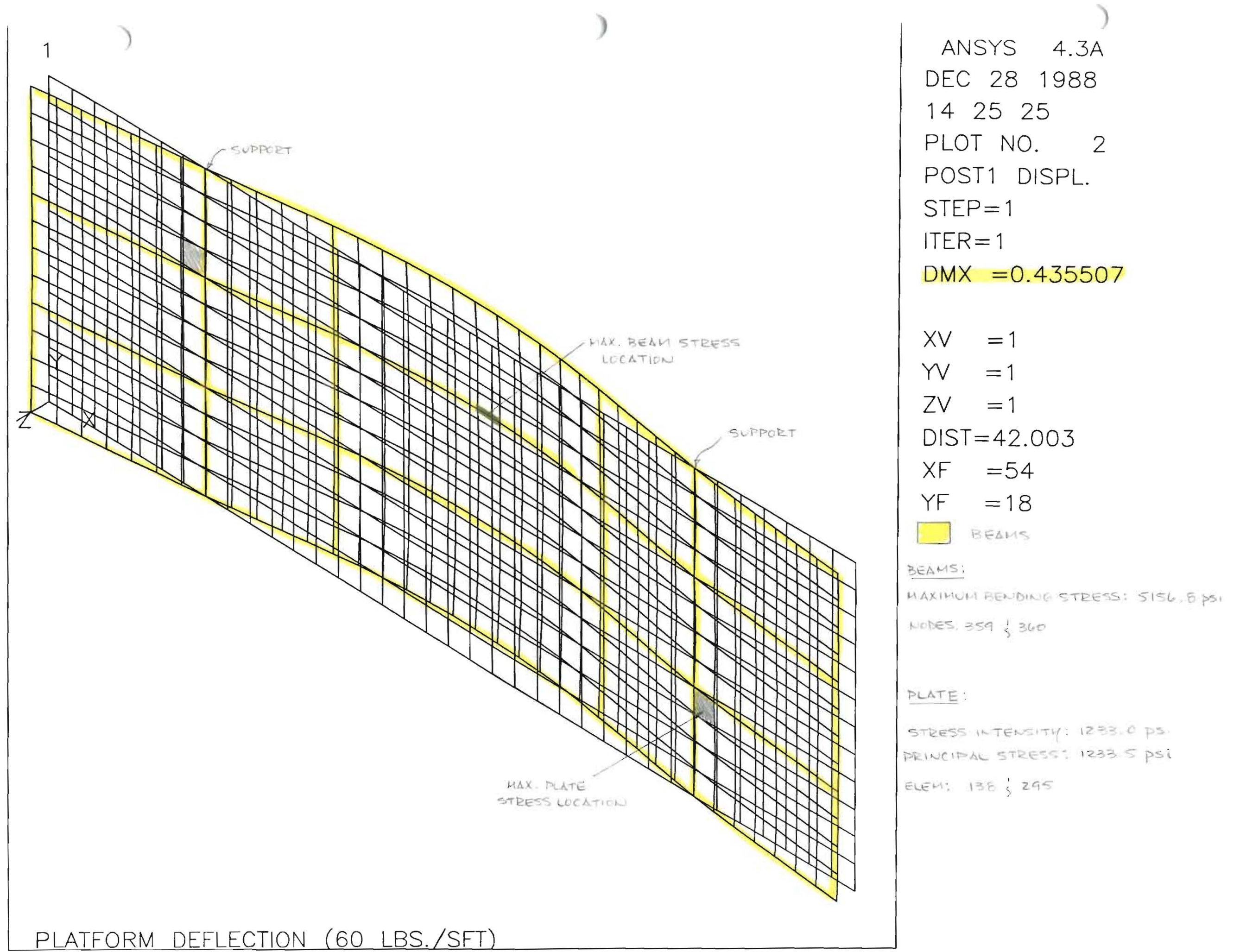


PRINT NODAL DISPLACEMENTS

***** POST1 NODAL DISPLACEMENT LISTING *****

LOAD STEP 1 ITERATION $=1$ SECTION $=1$

TIME $=0.00000 \mathrm{E}+00 \quad$ LOAD CASE $=1$

THE FOLLOWING $X, Y, Z$ DISPLACEMENTS ARE IN NODAL COORDINATES

\begin{tabular}{|c|c|c|c|c|c|c|}
\hline ODE & UX & UY & UZ & ROTX & ROTY & \\
\hline 1 & $0.00000000 E+00$ & $0.00000000 E+00$ & 343251 & $-0.19250116 \mathrm{E}-02$ & $0.10121088 \mathrm{E}-01$ & \\
\hline 2 & $00000000 E+00$ & $0.00000000 E+00$ & & & & \\
\hline 3 & $0000000 E+00$ & $0000000 E+00$ & & & & \\
\hline 4 & $000000 \mathrm{E}$ & $0.00000000 E+00$ & & & & \\
\hline 5 & 000000 & 0000000 & 0.1 & & & \\
\hline 6 & 0 & 0.0000000 & 0.11568525 & & & \\
\hline 7 & & 000000 & $35430 E-01$ & -0.229 & & \\
\hline 8 & & 000000 & E-01 & -0.2340 & & \\
\hline 9 & & 000000 & $0000000 \mathrm{~F}$ & $-0.23760252 \mathrm{E}$ & 01 & \\
\hline 10 & & 0000000 & & $-0.24016579 \mathrm{E}$ & $0.15902465 \mathrm{E}$ & \\
\hline 11 & & 00000000 & & $-0.24173530 \mathrm{E}$ & $0.16497079 \mathrm{E}$ & \\
\hline & & & & .02 & -01 & \\
\hline
\end{tabular}

***** POST1 NODAL DISPLACEMENT LISTING *****

LOAD STEP 1 ITERATION $=1$ SECTION $=1$

TIME $=0.00000 E+00 \quad$ LOAD CASE $=1$

THE FOLLOWING $X, Y, Z$ DISPLACEMENTS ARE IN NODAL COORDINATES

\begin{tabular}{|c|c|c|c|c|c|c|}
\hline NODE & UX & UY & UZ & ROTX & ROTY & \\
\hline 13 & $0.00000000 E+00$ & $0.00000000 E+00$ & -0.19335121 & $-0.24214836 \mathrm{E}-02$ & $0.16009560 \mathrm{E}-01$ & 0.0 \\
\hline 14 & $0.00000000 E+00$ & $0.00000000 E+00$ & -0.24001638 & $-0.24153895 \mathrm{E}-02$ & $0.15022410 \mathrm{E}-01$ & 0 \\
\hline 15 & $0.00000000 E+00$ & $0.00000000 E+00$ & -0.28223026 & $-0.24748365 \mathrm{E}-02$ & $0.13069641 \mathrm{E}-01$ & 0 \\
\hline 16 & $0.00000000 E+00$ & $0.00000000 E+00$ & -0.31814334 & $-0.25297513 \mathrm{E}-02$ & $0.10827056 \mathrm{E}-01$ & $\mathrm{c}$ \\
\hline 17 & $0.00000000 E+00$ & $0.00000000 E+00$ & -0.34694698 & $-0.25751533 \mathrm{E}-02$ & $0.83390663 \mathrm{E}-02$ & 0 \\
\hline 18 & $0.00000000 E+00$ & $0.00000000 E+00$ & -0.36799046 & $-0.26084706 \mathrm{E}-02$ & $0.56638267 \mathrm{E}-02$ & 0 \\
\hline 19 & $0.00000000 E+00$ & $0.00000000 E+00$ & -0.38080461 & $-0.26287003 E-02$ & $0.28632672 \mathrm{E}-02$ & 0 \\
\hline 20 & $0.00000000 E+00$ & $0.00000000 E+00$ & -0.38510735 & $-0.26354754 \mathrm{E}-02$ & $-0.23626886 \mathrm{E}-09$ & \\
\hline 21 & $0.00000000 E+00$ & $0.00000000 E+00$ & -0.38080461 & $-0.26287002 E-02$ & $-0.28632676 \mathrm{E}-02$ & \\
\hline 22 & $0.00000000 E+00$ & $0.00000000 E+00$ & -0.36799046 & $-0.26084704 \mathrm{E}-02$ & $-0.56638271 E-02$ & \\
\hline 23 & $0.00000000 E+00$ & $0.00000000 E+00$ & -0.34694698 & $-0.25751531 \mathrm{E}-02$ & $-0.83390666 \mathrm{E}-02$ & \\
\hline 24 & $0.00000000 E+00$ & $0.00000000 E+00$ & -0.31814334 & $-0.25297511 \mathrm{E}-02$ & $-0.10827056 \mathrm{E}-01$ & \\
\hline
\end{tabular}

***** POST1 NODAL DISPLACEMENT LISTING *****

LOAD STEP 1 ITERATION= 1 SECTION= 1

TIME $=0.00000 \mathrm{E}+00 \quad$ LOAD CASE $=1$

THE FOLLOWING $X, Y, Z$ DISPLACEMENTS ARE IN NODAL COORDINATES

$\begin{array}{ccccccc}\text { NODE } & \text { UX } & \text { UY } & \text { UZ } & \text { ROTX } & \text { ROTY } & F \\ 25 & 0.00000000 E+00 & 0.00000000 E+00 & -0.28223025 & -0.24748362 E-02 & -0.13069641 E-01 & 0.00 C \\ 26 & 0.00000000 E+00 & 0.00000000 E+00 & -0.24001638 & -0.24153891 E-02 & -0.15022410 E-01 & 0.00 C \\ 27 & 0.00000000 E+00 & 0.00000000 E+00 & -0.19335120 & -0.24214832 E-02 & -0.16009560 E-01 & 0.00 C \\ 28 & 0.00000000 E+00 & 0.00000000 E+00 & -0.14443955 & -0.24234587 E-02 & -0.16515140 E-01 & 0.00 C \\ 29 & 0.00000000 E+00 & 0.00000000 E+00 & -0.94783950 E-01 & -0.24173527 E-02 & -0.16497078 E-01 & 0.00 C \\ 30 & 0.00000000 E+00 & 0.00000000 E+00 & -0.46033730 E-01 & -0.24016575 E-02 & -0.15902465 E-01 & 0.00 C \\ 31 & 0.00000000 E+00 & 0.00000000 E+00 & 0.00000000 E+00 & -0.23760248 E-02 & -0.14677013 E-01 & 0.00 C\end{array}$


$0.41877634 \mathrm{E}-01-0.23403136 \mathrm{E}-02-0.13300874 \mathrm{E}-01$

$-0.12254973 \mathrm{E}-01$ $-0.22396377 \mathrm{E}-02-0.11486777 \mathrm{E}-01$

***** POST1 NODAL DISPLACEMENT LISTING *****

LOAD STEP 1 ITERATION $=1$ SECTION $=1$

TIME $=0.00000 \mathrm{E}+00 \quad$ LOAD CASE $=1$

THE FOLLOWING $X, Y, Z$ DISPLACEMENTS ARE IN NODAL COORDINATES

$\begin{array}{cccc}\text { NODE } & U X & U Y & U Z \\ 37 & 0.00000000 E+00 & 0.00000000 E+00 & 0.21280347 \\ 38 & 0.00000000 E+00 & 0.00000000 E+00 & 0.24343250 \\ 39 & 0.00000000 E+00 & 0.00000000 E+00 & 0.23808588 \\ 40 & 0.00000000 E+00 & 0.00000000 E+00 & 0.23369490 \\ 41 & 0.00000000 E+00 & 0.00000000 E+00 & 0.23032520 \\ 42 & 0.00000000 E+00 & 0.00000000 E+00 & 0.22790735 \\ 43 & 0.00000000 E+00 & 0.00000000 E+00 & 0.22636994 \\ 44 & 0.00000000 E+00 & 0.00000000 E+00 & 0.22583523 \\ 45 & 0.00000000 E+00 & 0.00000000 E+00 & 0.22636994 \\ 46 & 0.00000000 E+00 & 0.00000000 E+00 & 0.22790735 \\ 47 & 0.00000000 E+00 & 0.00000000 E+00 & 0.23032520 \\ 48 & 0.00000000 E+00 & 0.00000000 E+00 & 0.23369490\end{array}$

$\begin{array}{cc}\text { ROTX } & \text { ROTY } \\ -0.20142211 \mathrm{E}-02 & -0.10308247 \mathrm{E}-01 \\ 0.19250113 \mathrm{E}-02 & -0.10121088 \mathrm{E}-01 \\ -0.16301204 \mathrm{E}-02 & -0.10067694 \mathrm{E}-01 \\ -0.12932738 \mathrm{E}-02 & -0.10008731 \mathrm{E}-01 \\ -0.95736845 \mathrm{E}-03 & -0.99417443 \mathrm{E}-02 \\ -0.66348453 \mathrm{E}-03 & -0.98700313 \mathrm{E}-02 \\ -0.35247081 \mathrm{E}-03 & -0.98785536 \mathrm{E}-02 \\ -0.10276018 \mathrm{E}-10 & -0.98823441 \mathrm{E}-02 \\ 0.35247079 \mathrm{E}-03 & -0.98785536 \mathrm{E}-02 \\ 0.66348451 \mathrm{E}-03 & -0.98700313 \mathrm{E}-02 \\ 0.95736844 \mathrm{E}-03 & -0.99417443 \mathrm{E}-02 \\ 0.12932737 \mathrm{E}-02 & -0.10008731 \mathrm{E}-01\end{array}$

F

$0.00 \mathrm{C}$

0.00

***** POST1 NODAL DISPLACEMENT LISTING *****

LOAD STEP 1 ITERATION= 1 SECTION $=1$

TIME $=0.00000 \mathrm{E}+00 \quad$ LDAD CASE $=1$

THE FOLLOWING $X, Y, Z$ DISPLACEMENTS ARE IN NODAL COORDINATES

$\begin{array}{cccc}\text { NODE } & U X & U Y & U Z \\ 49 & 0.00000000 E+00 & 0.00000000 E+00 & 0.23808588 \\ 50 & 0.00000000 E+00 & 0.00000000 E+00 & 0.24343251 \\ 51 & 0.00000000 E+00 & 0.00000000 E+00 & 0.21280347 \\ 52 & 0.00000000 E+00 & 0.00000000 E+00 & 0.18151146 \\ 53 & 0.00000000 E+00 & 0.00000000 E+00 & 0.14928046 \\ 54 & 0.00000000 E+00 & 0.00000000 E+00 & 0.11568524 \\ 55 & 0.00000000 E+00 & 0.00000000 E+00 & 0.80135427 E-01 \\ 56 & 0.00000000 E+00 & 0.00000000 E+00 & 0.41877634 \mathrm{E}-01 \\ 57 & 0.00000000 \mathrm{E}+00 & 0.00000000 \mathrm{E}+00 & 0.00000000 \mathrm{E}+00 \\ 58 & 0.00000000 \mathrm{E}+00 & 0.00000000 \mathrm{E}+00 & -0.46033730 \mathrm{E}-01 \\ 59 & 0.00000000 \mathrm{E}+00 & 0.00000000 \mathrm{E}+00 & -0.94783950 \mathrm{E}-01 \\ 60 & 0.00000000 \mathrm{E}+00 & 0.00000000 \mathrm{E}+00 & -0.14443955\end{array}$

\begin{tabular}{cc} 
ROTX & \multicolumn{1}{c}{ ROTY } \\
$0.16301204 \mathrm{E}-02$ & $-0.10067694 \mathrm{E}-01$ \\
$0.19250116 \mathrm{E}-02$ & $0.10121088 \mathrm{E}-01$ \\
$0.20142211 \mathrm{E}-02$ & $-0.10308247 \mathrm{E}-01$ \\
$0.20986580 \mathrm{E}-02$ & $-0.10568002 \mathrm{E}-01$ \\
$0.21742726 \mathrm{E}-02$ & $-0.10942831 \mathrm{E}-01$ \\
$0.22396377 \mathrm{E}-02$ & $-0.11486777 \mathrm{E}-01$ \\
$0.22948547 \mathrm{E}-02$ & $-0.12254973 \mathrm{E}-01$ \\
$0.23403136 \mathrm{E}-02$ & $-0.13300874 \mathrm{E}-01$ \\
$0.23760249 \mathrm{E}-02$ & $-0.14677013 \mathrm{E}-01$ \\
$0.24016576 \mathrm{E}-02$ & $-0.15902465 \mathrm{E}-01$ \\
$0.24173527 \mathrm{E}-02$ & $-0.16497078 \mathrm{E}-01$ \\
$0.24234587 \mathrm{E}-02$ & $-0.16515140 \mathrm{E}-01$
\end{tabular}
0.006
0.006
0.000
0.000
0.000
0.000
0.006
0.000
0.000
0.006
0.000
0.006

\section{***** POST1 NODAL DISPLACEMENT LISTING *****}

LOAD STEP 1 ITERATION $=1$ SECTION $=1$

TIME $=0.00000 \mathrm{E}+00 \quad$ LOAD CASE $=1$

THE FOLLOWING $X, Y, Z$ DISPLACEMENTS ARE IN NODAL COORDINATES

$\begin{array}{cccc}\text { NODE } & \text { UX } & \text { UY } & \text { UZ } \\ 61 & 0.00000000 E+00 & 0.00000000 E+00 & -0.19335120 \\ 62 & 0.00000000 E+00 & 0.00000000 E+00 & -0.24001638 \\ 63 & 0.00000000 E+00 & 0.00000000 E+00 & -0.28223025\end{array}$
ROTX

$0.24214832 \mathrm{E}-02-0.16009560 \mathrm{E}-01$

$0.24153891 \mathrm{E}-02-0.15022410 \mathrm{E}-01$

$0.24748362 \mathrm{E}-02-0.13069641 \mathrm{E}-01$
0.006

0.000

0.006 
$64 \quad 0.00000000 E+00$

$\begin{array}{ll}65 & 0.00000000 \mathrm{E}+00\end{array}$

$66 \quad 0.00000000 E+00$

$67 \quad 0.00000000 E+00$

$\begin{array}{ll}68 & 0.00000000 E+00\end{array}$

$69 \quad 0.00000000 E+00$

$70 \quad 0.00000000 E+00$

$\begin{array}{ll}71 & 0.00000000 \mathrm{E}+00\end{array}$

$72 \quad 0.00000000 \mathrm{E}+00$
$0.00000000 E+00-0.31814334$

$0.00000000 E+00-0.34694698$

$0.00000000 \mathrm{E}+00-0.36799046$

$0.00000000 E+00-0.38080461$

$0.00000000 E+00-0.38510735$

$0.00000000 E+00-0.38080461$

$0.00000000 E+00=0.36799046$

$0.00000000 E+00-0.34694698$

$0.00000000 E+00-0.31814334$
$0.25297511 \mathrm{E}-02-0.10827057 \mathrm{E}-01$

$0.25751531 \mathrm{E}-02-0.83390666 \mathrm{E}-02$

$0.26084705 \mathrm{E}-02-0.56638271 \mathrm{E}-02$

$0.26287002 \mathrm{E}-02-0.28632676 \mathrm{E}-02$

$0.26354754 \mathrm{E}-02-0.23626691 \mathrm{E}-09$

$0.26287003 \mathrm{E}-02$

$0.26084706 \mathrm{E}-02$

$0.25751533 \mathrm{E}-02$

$0.25297514 \mathrm{E}-02$

$0.28632672 \mathrm{E}-02$

$0.56638267 \mathrm{E}-02$

$0.83390663 \mathrm{E}-02$

$0.10827056 \mathrm{E}-01$
0.006

0.006

0.006

0.00

0.00

0.00

0.000

0.000

0.000

\section{***** POST1 NODAL DISPLACEMENT LISTING *****}

LOAD STEP 1 ITERATION $=1$ SECTION $=1$

TIME $=0.00000 \mathrm{E}+00 \quad$ LOAD CASE $=1$

THE FOLLOWING $X, Y, Z$ DISPLACEMENTS ARE IN NODAL COORDINATES

$\begin{array}{ccccccc}\text { NDDE } & \text { UX } & \text { UY } & \text { UZ } & \text { ROTX } & \text { ROTY } & F \\ 73 & 0.00000000 E+00 & 0.00000000 E+00 & -0.28223026 & 0.24748365 E-02 & 0.13069641 E-01 & 0.006 \\ 74 & 0.00000000 E+00 & 0.00000000 E+00 & -0.24001638 & 0.24153895 E-02 & 0.15022410 E-01 & 0.006 \\ 75 & 0.00000000 E+00 & 0.00000000 E+00 & -0.19335121 & 0.24214836 E-02 & 0.16009561 E-01 & 0.006 \\ 76 & 0.00000000 E+00 & 0.00000000 E+00 & -0.14443955 & 0.24234590 E-02 & 0.16515140 E-01 & 0.006 \\ 77 & 0.00000000 E+00 & 0.00000000 E+00 & -0.94783953 E-01 & 0.24173531 E-02 & 0.16497079 E-01 & 0.006 \\ 78 & 0.00000000 E+00 & 0.00000000 E+00 & -0.46033731 E-01 & 0.24016579 E-02 & 0.15902466 E-01 & 0.006 \\ 79 & 0.00000000 E+00 & 0.00000000 E+00 & 0.00000000 E+00 & 0.23760252 E-02 & 0.14677013 E-01 & 0.006 \\ 80 & 0.00000000 E+00 & 0.00000000 E+00 & 0.41877635 E-01 & 0.23403140 E-02 & 0.13300875 E-01 & 0.006 \\ 81 & 0.00000000 E+00 & 0.00000000 E+00 & 0.80135430 E-01 & 0.22948551 E-02 & 0.12254973 E-01 & 0.006 \\ 82 & 0.00000000 E+00 & 0.00000000 E+00 & 0.11568525 & 0.22396381 E-02 & 0.11486778 E-01 & 0.006 \\ 83 & 0.00000000 E+00 & 0.00000000 E+00 & 0.14928046 & 0.21742730 E-02 & 0.10942831 E-01 & 0.006 \\ 84 & 0.00000000 E+00 & 0.00000000 E+00 & 0.18151147 & 0.20986583 E-02 & 0.10568003 E-01 & 0.006\end{array}$

***** POST1 NODAL DISPLACEMENT LISTING *****

LOAD STEP 1 ITERATION $=1$ SECTION= 1

TIME $=0.00000 E+00 \quad$ LOAD CASE $=1$

THE FOLLOWING $X, Y, Z$ DISPLACEMENTS ARE IN NODAL COORDINATES

$\begin{array}{cccc}\text { NODE } & \text { UX } & \text { UY } & U Z \\ 85 & 0.00000000 E+00 & 0.00000000 E+00 & 0.21280348 \\ 86 & 0.00000000 E+00 & 0.00000000 E+00 & 0.23808589 \\ 87 & 0.00000000 E+00 & 0.00000000 E+00 & 0.23369491 \\ 88 & 0.00000000 E+00 & 0.00000000 E+00 & 0.23032521 \\ 89 & 0.00000000 E+00 & 0.00000000 E+00 & 0.22790736 \\ 90 & 0.00000000 E+00 & 0.00000000 E+00 & 0.22636995 \\ 91 & 0.00000000 E+\infty 0 & 0.00000000 E+00 & 0.22583523 \\ 92 & 0.00000000 E+00 & 0.00000000 E+00 & 0.22636995 \\ 93 & 0.00000000++00 & 0.00000000 E+00 & 0.22790736 \\ 94 & 0.00000000 E+00 & 0.00000000 E+00 & 0.23032521 \\ 95 & 0.00000000 E+\infty 0 & 0.00000000 E+00 & 0.23369491 \\ 96 & 0.00000000 E+00 & 0.00000000 E+\infty 0 & 0.23808589\end{array}$

\begin{abstract}
ROTX
$0.20142214 E-02$

$0.16301208 \mathrm{E}-02$

$0.12932740 E-02$

$0.95736869 \mathrm{E}-03$

$0.66348470 E-03$

$0.35247088 \mathrm{E}-03$

$-0.10270196 \mathrm{E}-10$

$-0.35247090 \mathrm{E}-03$

$-0.66348471 E-03$

$-0.95736870 \mathrm{E}-03$

$-0.12932740 \mathrm{E}-02$

$-0.16301208 \mathrm{E}-02$
\end{abstract}

ROTY
$0.10308247 \mathrm{E}-01$
$0.10067694 \mathrm{E}-01$
$0.10008731 \mathrm{E}-01$
$0.99417450 \mathrm{E}-02$
$0.98700320 \mathrm{E}-02$
$0.98785544 \mathrm{E}-02$
$0.98823449 \mathrm{E}-02$
$0.98785544 \mathrm{E}-02$
$0.98700320 \mathrm{E}-02$
$0.99417450 \mathrm{E}-02$
$0.10008731 \mathrm{E}-01$
$0.10067694 \mathrm{E}-01$

0.000

0.000

0.000

0.000

0.000

0.000

0.00

0.006

0.006

0.000

0.006

0.006

LOAD STEP 1 ITERATION $=1$ SECTION $=1$

TIME $=0.00000 E+\infty \quad$ LOAD CASE $=1$

THE FOLLOWING $X, Y, Z$ DISPLACEMENTS ARE IN NODAL COORDINATES 


$\begin{array}{rcccc}\text { NODE } & U X & \text { UY } & U Z & \text { ROTX } \\ 97 & 0.00000000 E+00 & 0.00000000 E+00 & 0.20489208 & -0.26279438 E-02 \\ 98 & 0.00000000 E+00 & 0.00000000 \mathrm{E}+00 & 0.17106578 & -0.35279023 \mathrm{E}-02 \\ 99 & 0.00000000 \mathrm{E}+00 & 0.00000000 \mathrm{E}+00 & 0.13796763 & -0.38327897 \mathrm{E}-02 \\ 100 & 0.00000000 \mathrm{E}+00 & 0.00000000 \mathrm{E}+00 & 0.10442627 & -0.37355212 \mathrm{E}-02 \\ 101 & 0.00000000 \mathrm{E}+00 & 0.00000000 \mathrm{E}+00 & 0.69139360 \mathrm{E}-01 & -0.35414331 \mathrm{E}-02 \\ 102 & 0.00000000 \mathrm{E}+00 & 0.00000000 \mathrm{E}+00 & 0.30930459 \mathrm{E}-01 & -0.34507575 \mathrm{E}-02 \\ 103 & 0.00000000 \mathrm{E}+00 & 0.00000000 \mathrm{E}+00 & -0.11200437 \mathrm{E}-01 & -0.35219183 \mathrm{E}-02 \\ 104 & 0.00000000 \mathrm{E}+00 & 0.00000000 \mathrm{E}+00 & -0.57428078 \mathrm{E}-01 & -0.36542201 \mathrm{E}-02 \\ 105 & 0.00000000 \mathrm{E}+00 & 0.00000000 \mathrm{E}+00 & -0.10634125 & -0.37842931 \mathrm{E}-02 \\ 106 & 0.00000000 \mathrm{E}+00 & 0.00000000 \mathrm{E}+00 & -0.15549281 & -0.36324891 \mathrm{E}-02 \\ 107 & 0.00000000 \mathrm{E}+00 & 0.00000000 \mathrm{E}+00 & -0.20234551 & -0.29437156 \mathrm{E}-02 \\ 108 & 0.00000000 \mathrm{E}+00 & 0.00000000 \mathrm{E}+00 & -0.24689055 & -0.21411589 \mathrm{E}-02\end{array}$

$\begin{array}{ll}\text { ROTY } & \text { F } \\ 0.11420688 \mathrm{E}-01 & 0.00 C \\ 0.11121873 \mathrm{E}-01 & 0.00 C \\ 0.11029295 \mathrm{E}-01 & 0.006 \\ 0.11398770 \mathrm{E}-01 & 0.006 \\ 0.12183727 \mathrm{E}-01 & 0.006 \\ 0.13346266 \mathrm{E}-01 & 0.006 \\ 0.14760088 \mathrm{E}-01 & 0.006 \\ 0.15973911 \mathrm{E}-01 & 0.006 \\ 0.16491993 \mathrm{E}-01 & 0.006 \\ 0.16108976 \mathrm{E}-01 & 0.006 \\ 0.15055787 \mathrm{E}-01 & 0.00 C \\ 0.15217476 \mathrm{E}-01 & 0.006\end{array}$

***** POST1 NODAL DISPLACEMENT LISTING *****

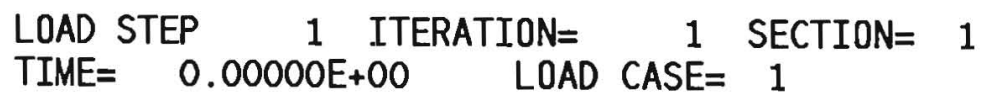

THE FOLLOWING $X, Y, Z$ DISPLACEMENTS ARE IN NODAL COORDINATES

$\begin{array}{lccc}\text { NODE } & \text { UX } & \text { UY } & U Z \\ 109 & 0.00000000 E+00 & 0.00000000 E+00 & -0.29228003 \\ 110 & 0.00000000 E+00 & 0.00000000 E+00 & -0.33153878 \\ 111 & 0.00000000 E+00 & 0.00000000 E+00 & -0.36216951 \\ 112 & 0.00000000 E+00 & 0.00000000 E+00 & -0.38406855 \\ 113 & 0.00000000 E+00 & 0.00000000 E+00 & -0.39726451 \\ 114 & 0.00000000 E+00 & 0.00000000 E+00 & -0.40167919 \\ 115 & 0.00000000 E+00 & 0.00000000 E+00 & -0.39726451 \\ 116 & 0.00000000 E+00 & 0.00000000 E+00 & -0.38406855 \\ 117 & 0.00000000 E+00 & 0.00000000 E+00 & -0.36216952 \\ 118 & 0.00000000 E+00 & 0.00000000 E+00 & -0.33153879 \\ 119 & 0.00000000 E+00 & 0.00000000 E+00 & -0.29228003 \\ 120 & 0.00000000 E+00 & 0.00000000 E+00 & -0.24689056\end{array}$

\begin{tabular}{cc} 
ROTX & \multicolumn{1}{c}{ ROTY } \\
$-0.35237681 \mathrm{E}-02$ & $0.14418014 \mathrm{E}-01$ \\
$-0.49434760 \mathrm{E}-02$ & $0.11694489 \mathrm{E}-01$ \\
$-0.58330056 \mathrm{E}-02$ & $0.87577306 \mathrm{E}-02$ \\
$-0.62875912 \mathrm{E}-02$ & $0.58552827 \mathrm{E}-02$ \\
$-0.65005156 \mathrm{E}-02$ & $0.29413845 \mathrm{E}-02$ \\
$-0.65639015 \mathrm{E}-02$ & $0.30460733 \mathrm{E}-09$ \\
$-0.65005156 \mathrm{E}-02$ & $-0.29413840 \mathrm{E}-02$ \\
$-0.62875912 \mathrm{E}-02$ & $-0.58552822 \mathrm{E}-02$ \\
$-0.58330055 \mathrm{E}-02$ & $-0.87577300 \mathrm{E}-02$ \\
$-0.49434760 \mathrm{E}-02$ & $-0.11694489 \mathrm{E}-01$ \\
$-0.35237681 \mathrm{E}-02$ & $-0.14418014 \mathrm{E}-01$ \\
$-0.21411586 \mathrm{E}-02$ & $-0.15217476 \mathrm{E}-01$
\end{tabular}

0.006

$0.00 C$

0.006

0.006

0.006

0.000

0.000

0.000

0.006

0.006

$0.00 C$

0.006

***** POST1 NODAL DISPLACEMENT LISTING *****

LOAD STEP 1 ITERATION= 1 SECTION= 1

TIME $=0.00000 E+00 \quad$ LOAD CASE $=1$

THE FOLLOWING $X, Y, Z$ DISPLACEMENTS ARE IN NODAL COORDINATES

$\begin{array}{lccc}\text { NODE } & U X & U Y & U Z \\ 121 & 0.00000000 E+00 & 0.00000000 E+00 & -0.20234552 \\ 122 & 0.00000000 E+00 & 0.00000000 E+00 & -0.15549283 \\ 123 & 0.00000000 E+00 & 0.00000000 E+00 & -0.10634127 \\ 124 & 0.00000000 E+00 & 0.00000000 E+00 & -0.57428095 E-01 \\ 125 & 0.00000000 E+00 & 0.00000000 E+00 & -0.11200454 E-01 \\ 126 & 0.00000000 E+00 & 0.00000000 E+00 & 0.30930443 E-01 \\ 127 & 0.00000000 E+00 & 0.00000000 E+00 & 0.69139342 E-01 \\ 128 & 0.00000000 E+00 & 0.00000000 E+00 & 0.10442625 \\ 129 & 0.00000000 E+00 & 0.00000000 E+00 & 0.13796761 \\ 130 & 0.00000000 E+00 & 0.00000000 E+00 & 0.17106575 \\ 131 & 0.00000000 E+00 & 0.00000000 E+00 & 0.20489205 \\ 132 & 0.00000000 E+00 & 0.00000000 E+00 & 0.19914771\end{array}$

$\begin{array}{cc}\text { ROTX } & \text { ROTY } \\ -0.29437151 E-02 & -0.15055787 E-01\end{array}$ $-0.36324885 \mathrm{E}-02-0.16108975 \mathrm{E}-01$ $-0.37842923 \mathrm{E}-02-0.16491992 \mathrm{E}-01$ $-0.36542192 \mathrm{E}-02-0.15973911 \mathrm{E}-01$ $-0.35219173 \mathrm{E}-02-0.14760088 \mathrm{E}-01$ $-0.34507566 \mathrm{E}-02-0.13346266 \mathrm{E}-01$ $-0.35414322 \mathrm{E}-02-0.12183726 \mathrm{E}-01$ $-0.37355204 \mathrm{E}-02-0.11398770 \mathrm{E}-01$ $-0.38327892 \mathrm{E}-02-0.11029295 \mathrm{E}-01$ $-0.35279021 \mathrm{E}-02-0.11121872 \mathrm{E}-01$ $-0.26279440 \mathrm{E}-02-0.11420688 \mathrm{E}-01$ $-0.10980163 \mathrm{E}-02$

$0.11928876 \mathrm{E}-01$ 0.006
0.006
0.006
0.006
0.006
0.006
0.006
0.006
0.006
0.006
0.006
0.006 ***** POST1 NODAL DISPLACEMENT LISTING ***** LOAD STEP 1 ITERATION= 1 SECTION $=1$ 
THE FOLLOWING $X, Y, Z$ DISPLACEMENTS ARE IN NODAL COORDINATES

$\begin{array}{lccc}\text { NODE } & U X & U Y & U Z \\ 133 & 0.00000000 E+00 & 0.00000000 E+00 & 0.16411797 \\ 134 & 0.00000000 E+00 & 0.00000000 E+00 & 0.13104066 \\ 135 & 0.00000000 E+00 & 0.00000000 E+00 & 0.98287190 E-01 \\ 136 & 0.00000000 E+00 & 0.00000000 E+00 & 0.63908667 E-01 \\ 137 & 0.00000000 E+00 & 0.00000000 E+00 & 0.26225208 E-01 \\ 138 & 0.00000000 E+00 & 0.00000000 E+00 & -0.16016816 E-01 \\ 139 & 0.00000000 E+00 & 0.00000000 E+00 & -0.62916154 \mathrm{E}-01 \\ 140 & 0.00000000 \mathrm{E}+00 & 0.00000000 \mathrm{E}+00 & -0.11279562 \\ 141 & 0.00000000 \mathrm{E}+00 & 0.00000000 \mathrm{E}+00 & -0.16252839 \\ 142 & 0.00000000 \mathrm{E}+00 & 0.00000000 \mathrm{E}+00 & -0.20890875 \\ 143 & 0.00000000 \mathrm{E}+00 & 0.00000000 \mathrm{E}+00 & -0.25272911 \\ 144 & 0.00000000 \mathrm{E}+00 & 0.00000000 \mathrm{E}+00 & -0.30088922\end{array}$

ROTX
$-0.78075562 \mathrm{E}-03$
$-0.38034274 \mathrm{E}-03$
$0.52891073 \mathrm{E}-04$
$0.44584574 \mathrm{E}-03$
$0.69826428 \mathrm{E}-03$
$0.69810482 \mathrm{E}-03$
$0.39210176 \mathrm{E}-03$
$-0.13733297 \mathrm{E}-03$
$-0.75215018 \mathrm{E}-03$
$-0.13236589 \mathrm{E}-02$
$-0.17351342 \mathrm{E}-02$
$-0.20703571 \mathrm{E}-02$

ROTY
$0.11292795 \mathrm{E}-01$

$0.10861354 \mathrm{E}-01$

$0.11093087 \mathrm{E}-01$

$0.11927596 \mathrm{E}-01$

$0.13270484 \mathrm{E}-01$

$0.14895790 \mathrm{E}-01$

$0.16269260 \mathrm{E}-01$

$0.16793089 \mathrm{E}-01$

$0.16163622 \mathrm{E}-01$

$0.14788337 \mathrm{E}-01$

$0.15413760 \mathrm{E}-01$

$0.15514458 \mathrm{E}-01$
0.006

0.006

0.006

0.006

0.00

0.006

0.00

0.000

0.000

0.00

0.00

0.000

***** POST1 NODAL DISPLACEMENT LISTING *****

LOAD STEP 1 ITERATION $=1$ SECTION $=1$

TIME $=0.00000 E+00 \quad$ LOAD CASE $=1$

THE FOLLOWING $X, Y, Z$ DISPLACEMENTS ARE IN NODAL COORDINATES

$\begin{array}{lccc}\text { NODE } & U X & U Y & U Z \\ 145 & 0.00000000 E+00 & 0.00000000 E+00 & -0.34334570 \\ 146 & 0.00000000 E+00 & 0.00000000 E+00 & -0.37614058 \\ 147 & 0.00000000 E+00 & 0.00000000 E+00 & -0.39931866 \\ 148 & 0.00000000 E+00 & 0.00000000 E+00 & -0.41319585 \\ 149 & 0.00000000 E+00 & 0.00000000 E+00 & -0.41782748 \\ 150 & 0.00000000 E+00 & 0.00000000 E+00 & -0.41319585 \\ 151 & 0.00000000 E+\infty 0 & 0.00000000 E+00 & -0.39931866 \\ 152 & 0.00000000 E+00 & 0.00000000 E+00 & -0.37614058 \\ 153 & 0.00000000 E+00 & 0.00000000 E+00 & -0.34334570 \\ 154 & 0.00000000 E+00 & 0.00000000 E+00 & -0.30088922 \\ 155 & 0.00000000 E+00 & 0.00000000 E+00 & -0.25272912 \\ 156 & 0.00000000 E+00 & 0.00000000 E+00 & -0.20890876\end{array}$

ROTX $-0.24791074 \mathrm{E}-02$ $-0.28760016 \mathrm{E}-02$ $-0.32012765 \mathrm{E}-02$ $-0.34136731 \mathrm{E}-02$ $-0.34873706 \mathrm{E}-02$ $-0.34136730 \mathrm{E}-02$ $-0.32012765 \mathrm{E}-02$ $-0.28760015 \mathrm{E}-02$ $-0.24791073 \mathrm{E}-02$ $-0.20703568 \mathrm{E}-02$ $-0.17351338 \mathrm{E}-02$ $-0.13236584 \mathrm{E}-02$

\section{ROTY}

$0.12566508 \mathrm{E}-01$ $0.93038991 \mathrm{E}-02$ $0.61684323 \mathrm{E}-02$ $0.30867094 \mathrm{E}-02$ $0.12750159 \mathrm{E}-09$ $-0.30867092 \mathrm{E}-02$ $-0.61684320 \mathrm{E}-02$ $-0.93038988 \mathrm{E}-02$ $-0.12566507 \mathrm{E}-01$ $-0.15514458 \mathrm{E}-01$ $-0.15413760 \mathrm{E}-01$ $-0.14788337 \mathrm{E}-01$
0.006

0.006

0.006

0.006

0.006

0.006

0.006

0.006

0.000

0.000

0.000

0.000

***** POST1 NODAL DISPLACEMENT LISTIING *****

$\begin{array}{lcccc}\text { LOAD STEP } & 1 & \text { ITERATION }= & 1 & \text { SECTION }=1 \\ \text { TIME }= & 0.00000 E+00 & \text { LOAD CASE }= & 1\end{array}$

THE FOLLOWING $X, Y, Z$ DISPLACEMENTS ARE IN NODAL COORDINATES

$\begin{array}{lccc}\text { NODE } & U X & U Y & U Z \\ 157 & 0.00000000 E+00 & 0.00000000 E+00 & -0.16252840 \\ 158 & 0.00000000 E+00 & 0.00000000 E+00 & -0.11279563 \\ 159 & 0.00000000 E+00 & 0.00000000 E+00 & -0.62916162 E-01 \\ 160 & 0.00000000 E+00 & 0.00000000 E+00 & -0.16016827 \mathrm{E}-01 \\ 161 & 0.00000000 \mathrm{E}+00 & 0.00000000 \mathrm{E}+00 & 0.26225197 \mathrm{E}-01 \\ 162 & 0.00000000 \mathrm{E}+00 & 0.00000000 \mathrm{E}+00 & 0.63908655 \mathrm{E}-01 \\ 163 & 0.00000000 \mathrm{E}+00 & 0.00000000 \mathrm{E}+00 & 0.98287176 \mathrm{E}-01 \\ 164 & 0.00000000 \mathrm{E}+00 & 0.00000000 \mathrm{E}+00 & 0.13104064 \\ 165 & 0.00000000 \mathrm{E}+00 & 0.00000000 \mathrm{E}+00 & 0.16411794 \\ 166 & 0.00000000 \mathrm{E}+00 & 0.00000000 \mathrm{E}+00 & 0.19914768 \\ 167 & 0.00000000 \mathrm{E}+00 & 0.00000000 \mathrm{E}+00 & 0.19815154 \\ 168 & 0.00000000 \mathrm{E}+00 & 0.00000000 \mathrm{E}+00 & 0.16606493\end{array}$

$0.00000000 E+00-0.16252840$

$0.00000000 E+00-0.11279563$

$0.00000000 E+00$

$0.00000000 E+00$

$0.00000000 E+00$

$0.00000000 E+\infty$

$0.00000000 \mathrm{E}+00$

$0.00000000 E+00$
ROTX $-0.75214950 \mathrm{E}-03$ $-0.13733210 \mathrm{E}-03$

$0.39210279 \mathrm{E}-03$

$0.69810593 \mathrm{E}-03$

$0.69826539 \mathrm{E}-03$

$0.44584678 \mathrm{E}-03$

$0.52891979 \mathrm{E}-04$

$-0.38034193 \mathrm{E}-03$

$-0.78075492 \mathrm{E}-03$

$-0.10980158 \mathrm{E}-02$

$0.30450520 \mathrm{E}-03$

$0.16470902 \mathrm{E}-02$
ROTY

$-0.16163621 \mathrm{E}-01$ $-0.16793089 \mathrm{E}-01$ $-0.16269260 \mathrm{E}-01$ $-0.14895790 E-01$ $-0.13270484 E-01$ $-0.11927596 \mathrm{E}-01$ $-0.11093087 \mathrm{E}-01$ $-0.10861354 \mathrm{E}-01$ $-0.11292794 \mathrm{E}-01$ $-0.11928875 \mathrm{E}-01$ $0.10949587 \mathrm{E}-01$ $0.10465583 \mathrm{E}-01$
0.006 0.006 0.006 0.006 0.006 0.00 0.006 0.006 0.006 0.00 0.006 0.006 
***** POST1 NODAL DISPLACEMENT LISTING *****

LOAD STEP 1 ITERATION $=1$ SECTION $=1$

$T I M E=0.00000 E+00 \quad$ LOAD CASE $=1$

THE FOLLOWING $X, Y, Z$ DISPLACEMENTS ARE IN NODAL COORDINATES

\begin{tabular}{|c|c|c|c|c|c|c|}
\hline NODE & UX & UY & UZ & ROTX & ROTY & \\
\hline 169 & $0.00000000 E+00$ & $0000000 E+00$ & 13517014 & $0.25372269 \mathrm{E}-02$ & $0.10258323 \mathrm{E}-01$ & 0.0 \\
\hline 170 & $000000 E+00$ & $000000 E+00$ & 0403332 & $0.30888685 \mathrm{E}-02$ & $0.10621259 \mathrm{E}-01$ & \\
\hline 171 & $000000 E+00$ & $000000 E+00$ & 0945337E-01 & $0.34950270 \mathrm{E}-02$ & $0.11558312 \mathrm{E}-01$ & \\
\hline 172 & +00 & $000000 E+00$ & $0.34172263 \mathrm{E}-01$ & $0.38065669 \mathrm{E}-02$ & $0.13078445 \mathrm{E}-01$ & \\
\hline 173 & $+\infty$ & $000000 E+00$ & $-0.79433365 E-02$ & $0.38938882 E-02$ & $0.15048206 \mathrm{E}$ & \\
\hline 174 & 2000000 & $0000000 E+00$ & $-0.55913188 \mathrm{E}-01$ & $0.35183753 \mathrm{E}-02$ & 0.16790921 & \\
\hline 175 & $0000000 E+00$ & $0.00000000 E+00$ & -0.10782527 & 0.28000900 & 0.17 & \\
\hline 176 & $0.00000000 E+00$ & $0.00000000 E+00$ & -0.16042184 & 0.16 & -01 & \\
\hline 177 & $0.00000000 E+00$ & $0.00000000 E+00$ & -0.21047467 & & & \\
\hline 178 & $0000000 E+00$ & $0.00000000 E+00$ & -0.2 & & & \\
\hline 179 & +00 & $0000000 E+00$ & -0.3 & & 0.15139524 & 0.006 \\
\hline .80 & OOOOOOOE & $0.00000000 E+00$ & -0.34606057 & $0.35982640 \mathrm{E}-03$ & $0.12455528 \mathrm{E}-01$ & 0.006 \\
\hline
\end{tabular}

***** POST1 NODAL DISPLACEMENT LISTING *****

LOAD STEP 1 ITERATION $=1$ SECTION= 1

TIME $=0.00000 \mathrm{E}+00 \quad$ LOAD CASE $=1$

THE FOLLOWING $X, Y, Z$ DISPLACEMENTS ARE IN NODAL COORDINATES

$\begin{array}{lccc}\text { NODE } & U X & U Y & U Z \\ 181 & 0.00000000 E+00 & 0.00000000 E+00 & -0.37891059 \\ 182 & 0.00000000 E+00 & 0.00000000 E+00 & -0.40263154 \\ 183 & 0.00000000 E+00 & 0.00000000 E+00 & -0.41701699 \\ 184 & 0.00000000 E+00 & 0.00000000 E+00 & -0.42184423 \\ 185 & 0.00000000 E+00 & 0.00000000 E+00 & -0.41701699 \\ 186 & 0.00000000 E+00 & 0.00000000 E+00 & -0.40263154 \\ 187 & 0.00000000 E+00 & 0.00000000 E+00 & -0.37891059 \\ 188 & 0.00000000 E+00 & 0.00000000 E+00 & -0.34606057 \\ 189 & 0.00000000 E+00 & 0.00000000 E+00 & -0.30450122 \\ 190 & 0.00000000 E+00 & 0.00000000 E+00 & -0.25725318 \\ 191 & 0.00000000 E+00 & 0.00000000 E+00 & -0.21047467 \\ 192 & 0.00000000 E+00 & 0.00000000 E+00 & -0.16042184\end{array}$

\begin{tabular}{cr} 
ROTX & \multicolumn{1}{c}{ ROTY } \\
$0.63363715 \mathrm{E}-03$ & $0.94349893 \mathrm{E}-02$ \\
$0.58074482 \mathrm{E}-03$ & $0.63591759 \mathrm{E}-02$ \\
$0.46257213 \mathrm{E}-03$ & $0.32091696 \mathrm{E}-02$ \\
$0.41122416 \mathrm{E}-03$ & $-0.11577574 \mathrm{E}-09$ \\
$0.46257216 \mathrm{E}-03$ & $-0.32091698 \mathrm{E}-02$ \\
$0.58074489 \mathrm{E}-03$ & $-0.63591761 \mathrm{E}-02$ \\
$0.63363724 \mathrm{E}-03$ & $-0.94349895 \mathrm{E}-02$ \\
$0.35982652 \mathrm{E}-03$ & $-0.12455528 \mathrm{E}-01$ \\
$-0.43183930 \mathrm{E}-03$ & $-0.15139524 \mathrm{E}-01$ \\
$-0.12808746 \mathrm{E}-02$ & $-0.15610049 \mathrm{E}-01$ \\
$0.14623632 \mathrm{E}-03$ & $-0.15995223 \mathrm{E}-01$ \\
$0.16946388 \mathrm{E}-02$ & $-0.17230950 \mathrm{E}-01$
\end{tabular}

0.000

0.006

0.006

0.006

0.006

0.006

0.000

0.006

0.006

0.006

0.006

0.000

***** POST1 NODAL DISPLACEMENT LISTING *****

LOAD STEP 1 ITERATION= 1 SECTION $=1$

TIME $=0.00000 \mathrm{E}+00 \quad$ LOAD CASE $=1$

THE FOLLOWING $X, Y, Z$ DISPLACEMENTS ARE IN NODAL COORDINATES
NODE
$\mathrm{UX}$
UY
UZ
ROTX
ROTY
193
$0.00000000 E+00$
$0.00000000 E+00-0.10782528$
$0.28000907 \mathrm{E}-02-0.17586865 \mathrm{E}-01$
$0.35183761 \mathrm{E}-02-0.16790921 \mathrm{E}-01$
$0.38938891 \mathrm{E}-02-0.15048205 \mathrm{E}-01$
$1950.00000000 \mathrm{E}+00$
$0.00000000 E+00-0.55913189 E-01$
$0.00000000 \mathrm{E}+00-0.79433413 \mathrm{E}-02$
$0.38065678 \mathrm{E}-02-0.13078445 \mathrm{E}-01$
$0.00000000 \mathrm{E}+00$
$0.00000000 E+00$
$0.34172258 \mathrm{E}-01$
$197 \quad 0.00000000 \mathrm{E}+00$
$0.00000000 E+00$
$0.70945329 \mathrm{E}-01$
$0.00000000 E+00$
$0.00000000 \mathrm{E}+00$
0.10403331
$199 \quad 0.00000000 \mathrm{E}+00$
$0.00000000 E+00$
0.13517012
200
$0.00000000 E+00$
$0.00000000 E+00$
0.16606491
201
$0.00000000 \mathrm{E}+00$
$0.00000000 E+00$
0.19815151
$0.34950278 \mathrm{E}-02-0.11558312 \mathrm{E}-01$
$0.30888693 \mathrm{E}-02-0.10621259 \mathrm{E}-01$
$0.25372276 \mathrm{E}-02-0.10258322 \mathrm{E}-01$
$0.16470912 \mathrm{E}-02-0.10465582 \mathrm{E}-01$
$0.30450633 \mathrm{E}-03-0.10949586 \mathrm{E}-01$
0.006
0.006
0.006
0.006
0.006
0.006
0.000
$0.00 C$
$0.00 C$ 
202

203

$0.00000000 E+00$

$0.00000000 \mathrm{E}+00$

204
$0.00000000 \mathrm{E}+00$

$0.00000000 E+00$

$0.00000000 E+00$
0.19860100

0.16964925

0.14051392
$-0.66920186 \mathrm{E}-03$

$-0.67524117 \mathrm{E}-03$

$-0.68214872 \mathrm{E}-03$
$0.96866673 E-02$

$0.96435675 \mathrm{E}-02$

$0.98265417 \mathrm{E}-02$
0.00

0.00

0.00

***** POST1 NODAL DISPLACEMENT LISTING *****

LOAD STEP 1 ITERATION $=1$ SECTION $=1$

TIME $=0.00000 \mathrm{E}+00 \quad$ LOAD CASE $=1$

THE FOLLOWING $X, Y, Z$ DISPLACEMENTS ARE IN NODAL COORDINATES

\begin{tabular}{|c|c|c|c|c|c|c|}
\hline JDE & UX & UY & UZ & ROTX & ROTY & \\
\hline & $0.00000000 E+00$ & $.00000000 E+00$ & 0.11035216 & $-0.69065072 E-03$ & $0.10347433 E-01$ & \\
\hline & & & $0.77973014 \mathrm{E}-0$ & & & \\
\hline & & & $4 \mathrm{E}-01$ & & & \\
\hline & & & & & & \\
\hline & & & $-0.2(x-1)$ & & & \\
\hline & & & & & & \\
\hline 211 & & & & & & \\
\hline 12 & & & & & & \\
\hline & & & & & & \\
\hline & & & & & & \\
\hline 11 & & & & & & \\
\hline & $0.00000000 E+00$ & $0.00000000 E+00$ & 007700100 & $-0.88877319 \mathrm{E}-03$ & $0.93792268 \mathrm{E}-02$ & \\
\hline
\end{tabular}

***** POST1 NODAL DISPLACEMENT LISTING *****

LOAD STEP 1 ITERATION $=1$ SECTION= 1

TIME $=0.00000 \mathrm{E}+00 \quad$ LOAD CASE $=1$

THE FOLLOWING $X, Y, Z$ DISPLACEMENTS ARE IN NODAL COORDINATES

\begin{tabular}{|c|c|c|c|}
\hline NODE & UX & UY & UZ \\
\hline 217 & $0.00000000 E+00$ & $0.00000000 E+00$ & -0.40082672 \\
\hline-218 & $0.00000000 \mathrm{E}+00$ & $0.00000000 E+00$ & -0.41543180 \\
\hline 1 & $0.00000000 E+00$ & $0.00000000 \mathrm{E}+00$ & -0.42035492 \\
\hline 220 & $0.00000000 E+00$ & $0.00000000 E+00$ & -0.41543180 \\
\hline 221 & $0.00000000 E+00$ & $0.00000000 E+00$ & -0.40082671 \\
\hline 222 & $0.00000000 \mathrm{E}+00$ & $0.00000000 \mathrm{E}+00$ & -0.37703428 \\
\hline 223 & $0.00000000 \mathrm{E}+00$ & $0.00000000 E+00$ & -0.34488358 \\
\hline 224 & $0.00000000 E+00$ & $0.00000000 E+00$ & -0.30553641 \\
\hline 225 & $0.00000000 \mathrm{E}+00$ & $0.00000000 E+00$ & -0.26045141 \\
\hline 226 & $0.00000000 \mathrm{E}+00$ & $0.00000000 \mathrm{E}+00$ & -0.21048531 \\
\hline 227 & $000000 E+00$ & $0000 E+00$ & -0.15690604 \\
\hline 228 & $0000 E+00$ & $000 \mathrm{E}+00$ & -0.10210566 \\
\hline
\end{tabular}

\begin{tabular}{cc} 
ROTX & \multicolumn{1}{c}{ ROTY } \\
$-0.89516684 \mathrm{E}-03$ & $0.64362949 \mathrm{E}-02$ \\
$-0.89923272 \mathrm{E}-03$ & $0.32729595 \mathrm{E}-02$ \\
$-0.90062771 \mathrm{E}-03$ & $-0.23084149 \mathrm{E}-09$ \\
$-0.89923269 \mathrm{E}-03$ & $-0.32729599 \mathrm{E}-02$ \\
$-0.89516677 \mathrm{E}-03$ & $-0.64362953 \mathrm{E}-02$ \\
$-0.88877309 \mathrm{E}-03$ & $-0.93792272 \mathrm{E}-02$ \\
$-0.88057265 \mathrm{E}-03$ & $-0.11989744 \mathrm{E}-01$ \\
$-0.87117480 \mathrm{E}-03$ & $-0.14159815 \mathrm{E}-01$ \\
$-0.86116317 \mathrm{E}-03$ & $-0.15805152 \mathrm{E}-01$ \\
$-0.83283178 \mathrm{E}-03$ & $-0.17383514 \mathrm{E}-01$ \\
$-0.80495775 \mathrm{E}-03$ & $-0.18203913 \mathrm{E}-01$ \\
$-0.77840620 \mathrm{E}-03$ & $-0.18179946 \mathrm{E}-01$
\end{tabular}

0.006

0.006

0.006

0.006

0.006

$0: 006$

0.006

0.006

0.006

0.006

0.006

0.006

***** POST1 NODAL DISPLACEMENT LISTING *****

LOAD STEP 1 ITERATION $=1$ SECTION $=1$

TIME $=0.00000 \mathrm{E}+00 \quad$ LOAD CASE $=1$

THE FOLLOWING $X, Y, Z$ DISPLACEMENTS ARE IN NODAL COORDINATES
NODE
229
$\mathrm{UX}$
UY
$\mathrm{UZ}$
ROTX
ROTY
230
$0.00000000 E+00$
$0.00000000 E+00$
$-0.48784209 \mathrm{E}-0$
$-0.75416049 \mathrm{E}-03$
$-0.17197891 \mathrm{E}-01$
$0.00000000 \mathrm{E}+00$
$0.00000000 E+00$
$0.00000000 \mathrm{E}+00$
$-0.73304872 \mathrm{E}-03-0.15133697 \mathrm{E}-01$
231
$0.00000000 \mathrm{E}+00$
$0.00000000 E+00$
$0.41827251 \mathrm{E}-01$
$0.77973009 \mathrm{E}-01$
$-0.71551826 \mathrm{E}-03-0.12878749 \mathrm{E}-01$
$0.00000000 \mathrm{E}+00$
0.11035215
$-0.70153690 \mathrm{E}-03$
$-0.69065052 \mathrm{E}-03$
$-0.11324720 \mathrm{E}-01$
$0.00000000 E+\infty 0$
$0.00000000 E+00$
$0.00000000+00$
$-0.69065052 \mathrm{E}-03-0.10347432 \mathrm{E}-01$
0.000
0.000
0.006
$0.00 \mathrm{C}$
0.006 


$\begin{array}{llll}234 & 0.00000000 E+00 & 0.00000000 E+00 & 0.14051391 \\ 235 & 0.00000000 E+00 & 0.00000000 E+00 & 0.16964923 \\ 236 & 0.00000000 E+00 & 0.00000000 E+00 & 0.19860098 \\ 237 & 0.00000000 E+00 & 0.00000000 E+00 & 0.19441296 \\ 238 & 0.00000000 E+00 & 0.00000000 E+00 & 0.16272245 \\ 239 & 0.00000000 E+00 & 0.00000000 E+00 & 0.13234889 \\ 240 & 0.00000000 E+00 & 0.00000000 E+00 & 0.10176792\end{array}$

$-0.68214852 \mathrm{E}-03-0.98265410 \mathrm{E}-02$ $-0.67524098 \mathrm{E}-03-0.96435666 \mathrm{E}-02$ $-0.66920167 \mathrm{E}-03-0.96866664 \mathrm{E}-02$ $-0.14690125 \mathrm{E}-02$ $-0.25672075 \mathrm{E}-02$ $-0.31459507 \mathrm{E}-02$ $-0.33655118 \mathrm{E}-02$
$0.10843421 \mathrm{E}-01$

$0.10302595 \mathrm{E}-01$

$0.10066687 \mathrm{E}-01$

$0.10434728 \mathrm{E}-01$
0.006

0.006

0.006

0.006

0.006

0.00

0.006

***** POST1 NODAL DISPLACEMENT LISTING *****

LOAD STEP 1 ITERATION= 1 SECTION $=1$

TIME $=0.00000 \mathrm{E}+00 \quad$ LOAD CASE $=1$

THE FOLLOWING $X, Y, Z$ DISPLACEMENTS ARE IN NODAL COORDINATES
NODE
UX
UY
UZ
ROTX
ROTY
$241 \quad 0.00000000 E+00$
$0.00000000 E+00$
$0.69159283 \mathrm{E}-01-0.34807505 \mathrm{E}-02$
$0.11420437 \mathrm{E}-01$
$0.13039268 \mathrm{E}-01$
$0.15142696 \mathrm{E}-01$
$0.17013042 \mathrm{E}-01$
$0.00000000 E+00$
$0.32650282 \mathrm{E}-01-0.36205538 \mathrm{E}-02$
$2430.00000000 \mathrm{E}+00$
$0.00000000 \mathrm{E}+00$
$-0.95460161 E-02$
$-0.57985490 E-01$
$-0.37290346 \mathrm{E}-02$
$0.00000000 E+00-0.11067634$
$-0.32860294 \mathrm{E}-02$
$245 \quad 0.00000000 E+00$
$0.00000000 E+00-0.16418818$
$-0.26667442 \mathrm{E}-02$
$247 \quad 0.00000000 \mathrm{E}+00$
$0.00000000 E+00-0.21508462$
$-0.15713356 \mathrm{E}-02$
$0.00000000 E+00-0.26247766$
$-0.47179422 \mathrm{E}-03$
$0.17888773 \mathrm{E}-01$
$0.17544052 \mathrm{E}-01$
$0.16248613 \mathrm{E}-01$
$0.15806591 \mathrm{E}-01$
$-0.15812150 \mathrm{E}-02$
$249 \quad 0.00000000 \mathrm{E}+00$
$0.00000000 E+00-0.31020358$
250
$0.00000000 E+00-0.35228467$
$-0.26752592 \mathrm{E}-02$
$0.15307955 \mathrm{E}-01$
$0.12642128 \mathrm{E}-01$
$251 \quad 0.00000000 \mathrm{E}+00$
$0.00000000 E+00-0.38566564$
$-0.32476984 \mathrm{E}-02$
$0.96073654 \mathrm{E}-02$
$0.00000000 E+00-0.40983071$
$-0.34432776 \mathrm{E}-02$
$0.64884685 \mathrm{E}-02$
0.000
0.000
0.000
0.006
0.006
0.000
0.006
0.000
0.006
0.006
0.006
0.006

***** POST1 NODAL DISPLACEMENT LISTING *****

LOAD STEP 1 ITERATION $=1$ SECTION $=1$

TIME $=0.00000 \mathrm{E}+00 \quad$ LOAD CASE $=1$

THE FOLLOWING $X, Y, Z$ DISPLACEMENTS ARE IN NODAL COORDINATES

$\begin{array}{lc}\text { NODE } & U X \\ 253 & 0.00000000 E+00 \\ 254 & 0.00000000 E+00 \\ 255 & 0.00000000 E+00 \\ 256 & 0.00000000 E+00 \\ 257 & 0.00000000 E+00 \\ 258 & 0.00000000 E+00 \\ 259 & 0.00000000 E+00 \\ 260 & 0.0000000 E+00 \\ 261 & 0.00000000 E+00 \\ 262 & 0.00000000 E+00 \\ 263 & 0.00000000 E+00 \\ 264 & 0.00000000 E+00\end{array}$

\section{UY}

$0.00000000 E+\infty 0-0.42450710$

$0.00000000 E+00-0.42943529$

$0.00000000 \mathrm{E}+00-0.42450710$

$0.00000000 \mathrm{E}+00-0.40983070$

$0.00000000 E+00-0.38566564$

$0.00000000 \mathrm{E}+00-0.35228467$

$0.00000000 \mathrm{E}+00-0.31020358$

$0.00000000 E+00-0.26247765$

$0.00000000 E+00-0.21508461$

$0.00000000 E+00-0.16418818$

$0.00000000 E+00-0.11067633$

$0.00000000 E+00-0.57985486 E-01$
ROTX

$-0.34881759 \mathrm{E}-02$

$-0.34934837 \mathrm{E}-02$

$-0.34881758 \mathrm{E}-02$

$-0.34432776 \mathrm{E}-02$

$-0.32476983 \mathrm{E}-02$

$-0.26752592 \mathrm{E}-02$

$-0.15812148 \mathrm{E}-02$

$-0.47179411 \mathrm{E}-03$

$-0.15713354 \mathrm{E}-02$

$-0.26667441 \mathrm{E}-02$

$-0.32860293 \mathrm{E}-02$

$-0.35931111 \mathrm{E}-02$
ROTY

$0.32779387 \mathrm{E}-02$

$-0.34763270 E-09$

$-0.32779394 E-02$

$-0.64884692 E-02$

$-0.96073661 E-02$

$-0.12642128 E-01$

$-0.15307955 E-01$

$-0.15806592 E-01$

$-0.16248613 E-01$

$-0.17544052 E-01$

$-0.17888773 E-01$

$-0.17013042 E-01$
0.006

0.000

0.006

0.006

0.006

0.006

0.006

0.006

0.006

0.006

0.000

0.006

***** POST1 NODAL DISPLACEMENT LISTING *****

LOAD STEP 1 ITERATION= 1 SECTION= 1

TIME $=0.00000 \mathrm{E}+00 \quad$ LOAD CASE $=1$

THE FOLLOWING $X, Y, Z$ DISPLACEMENTS ARE IN NODAL COORDINATES

NODE

265
UX

$0.00000000 E+00$
UY

$0.00000000 \mathrm{E}+\infty \quad-0.95460141 \mathrm{E}-02$
ROTX

ROTY

$-0.37290345 E-02-0.15142695 E-01$ 


\begin{tabular}{|c|c|c|c|c|c|c|}
\hline 266 & $0.00000000 E+00$ & $0.00000000 E+00$ & $0.32650282 \mathrm{E}-01$ & $-0.36205537 \mathrm{E}-02$ & $-0.13039268 \mathrm{E}-01$ & 0.006 \\
\hline 267 & $0.00000000 E+00$ & $0.00000000 E+00$ & $0.69159279 E-01$ & $-0.34807504 E-02$ & $-0.11420436 \mathrm{E}-01$ & 0.006 \\
\hline 268 & $0.00000000 E+00$ & $0.00000000 E+00$ & 0.10176791 & $3655118 \mathrm{E}-02$ & $-0.10434727 \mathrm{E}-01$ & 0.006 \\
\hline 269 & $0.00000000 E+00$ & $0.00000000 E+00$ & 0.13234888 & 459507E-02 & $-0.10066686 \mathrm{E}-01$ & 0.006 \\
\hline 270 & $0.00000000 E+00$ & $0.00000000 E+00$ & 0.16272243 & 2079E-02 & $-0.10302594 \mathrm{E}-01$ & 0.006 \\
\hline 271 & $0000000 E+00$ & $0.00000000 E+00$ & 0.19 & $0131 E-02$ & $-0.10843420 E-01$ & 0.006 \\
\hline 272 & $0000000 E+00$ & $0.00000000 E+00$ & 0.192 & $54572 \mathrm{E}-10$ & $0.11576214 \mathrm{E}-01$ & 0.000 \\
\hline 273 & $000000 E+00$ & $0.00000000 E+00$ & 0.15830596 & $24020 E-10$ & $0.10790134 \mathrm{E}-01$ & 0.006 \\
\hline 274 & $2000000 E+00$ & $0.00000000 E+00$ & 0.12688099 & $-0.34388191 E-10$ & $0.10277149 \mathrm{E}-01$ & 0.006 \\
\hline 275 & $0.00000000 E+00$ & $0.00000000 E+00$ & $0.95896157 \mathrm{E}-01$ & $-0.43433221 \mathrm{E}-10$ & $0.10518284 E-01$ & 0.006 \\
\hline 276 & $0.00000000 \mathrm{E}+00$ & $0.00000000 E+00$ & $0.63077900 \mathrm{E}-01$ & $0.63324836 \mathrm{E}-10$ & $0.11487972 \mathrm{E}-01$ & 0.006 \\
\hline
\end{tabular}

***** POST1 NODAL DISPLACEMENT LISTING *****

LOAD STEP 1 ITERATION $=1$ SECTION $=1$

TIME $=0.00000 \mathrm{E}+00 \quad$ LOAD CASE $=1$

THE FOLLOWING $X, Y, Z$ DISPLACEMENTS ARE IN NODAL COORDINATES

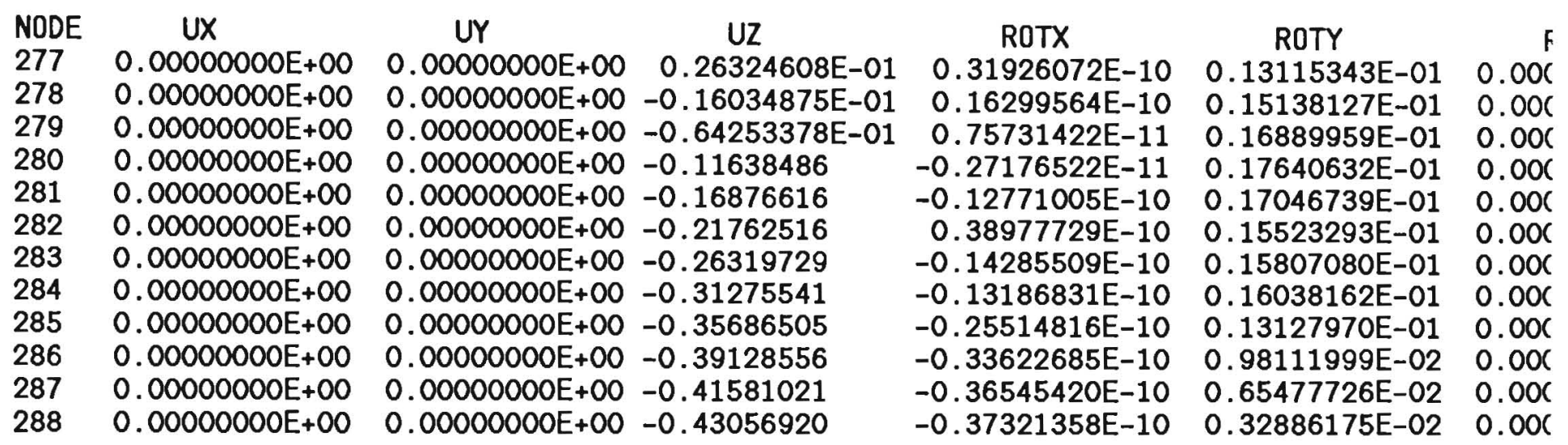

***** POST1 NODAL DISPLACEMENT LISTING *****

LOAD STEP 1 ITERATION $=1$ SECTION $=1$

TIME $=0.00000 E+00 \quad$ LOAD CASE $=1$

THE FOLLOWING $X, Y, Z$ DISPLACEMENTS ARE IN NODAL COORDINATES

$\begin{array}{lcccccc}\text { NODE } & U X & U Y & U Z & \text { ROTX } & \text { ROTY } & F \\ 289 & 0.00000000 E+00 & 0.00000000 E+00 & -0.43550697 & -0.37487541 E-10 & -0.35707292 \mathrm{E}-09 & 0.006 \\ 290 & 0.00000000 \mathrm{E}+00 & 0.00000000 \mathrm{E}+00 & -0.43056920 & -0.37321062 \mathrm{E}-10 & -0.32886182 \mathrm{E}-02 & 0.006 \\ 291 & 0.00000000 \mathrm{E}+00 & 0.00000000 \mathrm{E}+00 & -0.41581021 & -0.36544894 \mathrm{E}-10 & -0.65477733 \mathrm{E}-02 & 0.006 \\ 292 & 0.00000000 \mathrm{E}+00 & 0.00000000 \mathrm{E}+00 & -0.39128555 & -0.33621987 \mathrm{E}-10 & -0.98112006 \mathrm{E}-02 & 0.006 \\ 293 & 0.00000000 \mathrm{E}+00 & 0.00000000 \mathrm{E}+00 & -0.35686504 & -0.25514107 \mathrm{E}-10 & -0.13127971 \mathrm{E}-01 & 0.006 \\ 294 & 0.00000000 \mathrm{E}+00 & 0.00000000 \mathrm{E}+00 & -0.31275540 & -0.13186267 \mathrm{E}-10 & -0.16038162 \mathrm{E}-01 & 0.006 \\ 295 & 0.00000000 \mathrm{E}+00 & 0.00000000 \mathrm{E}+00 & -0.26319728 & -0.14285211 \mathrm{E}-10 & -0.15807080 \mathrm{E}-01 & 0.006 \\ 296 & 0.00000000 \mathrm{E}+00 & 0.00000000 \mathrm{E}+00 & -0.21762515 & 0.38977611 \mathrm{E}-10 & -0.15523294 \mathrm{E}-01 & 0.006 \\ 297 & 0.00000000 \mathrm{E}+00 & 0.00000000 \mathrm{E}+00-0.16876615 & -0.12771441 \mathrm{E}-10 & -0.17046739 \mathrm{E}-01 & 0.006 \\ 298 & 0.00000000 \mathrm{E}+00 & 0.00000000 \mathrm{E}+00-0.11638485 & -0.27178527 \mathrm{E}-11 & -0.17640632 \mathrm{E}-01 & 0.006 \\ 299 & 0.00000000 \mathrm{E}+00 & 0.00000000 \mathrm{E}+00-0.64253373 \mathrm{E}-01 & 0.75738325 \mathrm{E}-11 & -0.16889958 \mathrm{E}-01 & 0.006 \\ 300 & 0.00000000 \mathrm{E}+00 & 0.00000000 \mathrm{E}+00-0.16034873 \mathrm{E}-01 & 0.16301968 \mathrm{E}-10 & -0.15138127 \mathrm{E}-01 & 0.006\end{array}$

***** POST1 NODAL DISPLACEMENT LISTING *****

LOAD STEP 1 ITERATION $=1$ SECTION $=1$

TIME $=0.00000 \mathrm{E}+00 \quad$ LOAD CASE $=1$ 
THE FOLLOWING $X, Y, Z$ DISPLACEMENTS ARE IN NODAL COORDINATES

$\begin{array}{lccccccc}\text { NODE } & \text { UX } & \text { UY } & \text { UZ } & \text { ROTX } & \text { ROTY } & F \\ 301 & 0.00000000 E+00 & 0.00000000 E+00 & 0.26324608 E-01 & 0.31930019 E-10 & -0.13115343 E-01 & 0.006 \\ 302 & 0.00000000 E+00 & 0.00000000 E+00 & 0.63077896 E-01 & 0.63328368 E-10 & -0.11487972 E-01 & 0.006 \\ 303 & 0.00000000 E+00 & 0.00000000 E+00 & 0.95896150 E-01 & -0.43426843 E-10 & -0.10518284 E-01 & 0.006 \\ 304 & 0.00000000 E+00 & 0.00000000 E+00 & 0.12688098 & -0.34347806 E-10 & -0.10277148 E-01 & 0.006 \\ 305 & 0.00000000 E+00 & 0.00000000 E+00 & 0.15830594 & -0.33464104 E-10 & -0.10790133 E-01 & 0.00 C \\ 306 & 0.00000000 E+00 & 0.00000000 E+00 & 0.19203317 & -0.18467351 E-10 & -0.11576213 E-01 & 0.00 C \\ 307 & 0.00000000 E+00 & 0.00000000 E+00 & 0.19441296 & 0.14690124 E-02 & 0.10843421 E-01 & 0.00 C \\ 308 & 0.00000000 E+00 & 0.00000000 E+00 & 0.16272245 & 0.25672074 E-02 & 0.10302595 E-01 & 0.006 \\ 309 & 0.00000000 E+00 & 0.00000000 E+00 & 0.13234889 & 0.31459506 E-02 & 0.10066687 E-01 & 0.00 C \\ 310 & 0.00000000 E+00 & 0.00000000 E+00 & 0.10176792 & 0.33655118 E-02 & 0.10434728 E-01 & 0.00 C \\ 311 & 0.00000000 E+00 & 0.00000000 E+00 & 0.69159283 E-01 & 0.34807505 E-02 & 0.11420437 E-01 & 0.00 C \\ 312 & 0.00000000 E+00 & 0.00000000 E+00 & 0.32650282 E-01 & 0.36205538 E-02 & 0.13039268 E-01 & 0.00 C\end{array}$

***** POST1 NODAL DISPLACEMENT LISTING *****

LOAD STEP 1 ITERATION $=1$ SECTION $=1$

TIME $=0.00000 \mathrm{E}+00 \quad$ LOAD CASE $=1$

THE FOLLOWING $X, Y, Z$ DISPLACEMENTS ARE IN NODAL COORDINATES

$\begin{array}{lccc}\text { NODE } & U X & U Y & U Z \\ 313 & 0.00000000 E+00 & 0.00000000 E+00 & -0.95460160 E-02 \\ 314 & 0.00000000 E+00 & 0.00000000 E+00 & -0.57985490 E-01 \\ 315 & 0.00000000 E+00 & 0.00000000 E+00 & -0.11067634 \\ 316 & 0.00000000 E+00 & 0.00000000 E+00 & -0.16418818 \\ 317 & 0.00000000 E+00 & 0.00000000 E+00 & -0.21508462 \\ 318 & 0.00000000 E+00 & 0.00000000 E+00 & -0.26247766 \\ 319 & 0.00000000 E+00 & 0.00000000 E+00 & -0.31020358 \\ 320 & 0.00000000 E+00 & 0.00000000 E+00 & -0.35228467 \\ 321 & 0.00000000 E+00 & 0.00000000 E+00 & -0.38566564 \\ 322 & 0.00000000 E+00 & 0.00000000 E+00 & -0.40983071 \\ 323 & 0.00000000 E+00 & 0.00000000 E+00 & -0.42450710 \\ 324 & 0.00000000 E+00 & 0.00000000 E+00 & -0.42943529\end{array}$

ROTX
$0.37290346 \mathrm{E}-02$
$0.35931112 \mathrm{E}-02$
$0.32860294 \mathrm{E}-02$
$0.26667442 \mathrm{E}-02$
$0.15713356 \mathrm{E}-02$
$0.47179418 \mathrm{E}-03$
$0.15812149 \mathrm{E}-02$
$0.26752592 \mathrm{E}-02$
$0.32476983 \mathrm{E}-02$
$0.34432776 \mathrm{E}-02$
$0.34881758 \mathrm{E}-02$
$0.34934837 \mathrm{E}-02$

ROTY
$0.15142696 \mathrm{E}-01$

$0.17013042 \mathrm{E}-01$

$0.17888773 \mathrm{E}-01$

$0.17544052 \mathrm{E}-01$

$0.16248613 \mathrm{E}-01$

$0.15806591 \mathrm{E}-01$

$0.15307955 \mathrm{E}-01$

$0.12642128 \mathrm{E}-01$

$0.96073654 \mathrm{E}-02$

$0.64884685 \mathrm{E}-02$

$0.32779387 \mathrm{E}-02$

$-0.34763297 \mathrm{E}-09$

0.006

0.006

0.006

0.006

0.006

0.006

0.006

0.006

0.006

0.006

0.006

0.006

\begin{abstract}
***** POST1 NODAL DISPLACEMENT LISTING *****
\end{abstract}
LOAD STEP 1 ITERATION= $\begin{gathered}1 \\ \text { TIME }=0.00000 E+00 \quad \text { SECTION }=1 \\ \text { LOAD }\end{gathered}$

THE FOLLOWING $X, Y, Z$ DISPLACEMENTS ARE IN NODAL COORDINATES

$\begin{array}{lccc}\text { NODE } & U X & U Y & U Z \\ 325 & 0.00000000 \mathrm{E}+00 & 0.00000000 \mathrm{E}+00 & -0.42450710 \\ 326 & 0.00000000 \mathrm{E}+00 & 0.00000000 \mathrm{E}+00 & -0.40983070 \\ 327 & 0.00000000 \mathrm{E}+00 & 0.00000000 \mathrm{E}+00 & -0.38566564 \\ 328 & 0.00000000 \mathrm{E}+00 & 0.00000000 \mathrm{E}+00 & -0.35228467 \\ 329 & 0.00000000 \mathrm{E}+00 & 0.00000000 \mathrm{E}+00 & -0.31020358 \\ 330 & 0.00000000 \mathrm{E}+00 & 0.00000000 \mathrm{E}+00 & -0.26247765 \\ 331 & 0.00000000 \mathrm{E}+00 & 0.00000000 \mathrm{E}+00 & -0.21508461 \\ 332 & 0.00000000 \mathrm{E}+00 & 0.00000000 \mathrm{E}+00 & -0.16418818 \\ 333 & 0.00000000 \mathrm{E}+00 & 0.00000000 \mathrm{E}+00 & -0.11067633 \\ 334 & 0.00000000 \mathrm{E}+00 & 0.00000000 \mathrm{E}+00 & -0.57985486 \mathrm{E}-01 \\ 335 & 0.00000000 \mathrm{E}+00 & 0.00000000 \mathrm{E}+00 & -0.95460140 \mathrm{E}-02 \\ 336 & 0.00000000 \mathrm{E}+00 & 0.00000000 \mathrm{E}+00 & 0.32650282 \mathrm{E}-01\end{array}$

$\begin{array}{cc}\text { ROTX } & \text { ROTY } \\ 0.34881758 \mathrm{E}-02 & -0.32779394 \mathrm{E}-02 \\ 0.34432775 \mathrm{E}-02 & -0.64884692 \mathrm{E}-02 \\ 0.32476983 \mathrm{E}-02 & -0.96073661 \mathrm{E}-02 \\ 0.26752591 \mathrm{E}-02 & -0.12642128 \mathrm{E}-01 \\ 0.15812148 \mathrm{E}-02 & -0.15307955 \mathrm{E}-01 \\ 0.47179408 \mathrm{E}-03 & -0.15806592 \mathrm{E}-01 \\ 0.15713355 \mathrm{E}-02 & -0.16248613 \mathrm{E}-01 \\ 0.26667441 \mathrm{E}-02 & -0.17544052 \mathrm{E}-01 \\ 0.32860293 \mathrm{E}-02 & -0.17888773 \mathrm{E}-01 \\ 0.35931111 \mathrm{E}-02 & -0.17013042 \mathrm{E}-01 \\ 0.37290345 \mathrm{E}-02 & -0.15142695 \mathrm{E}-01 \\ 0.36205537 \mathrm{E}-02 & -0.13039268 \mathrm{E}-01\end{array}$

0.006

0.000

0.006

0.00

0.00

0.006

0.006

0.006

0.006

0.006

0.006

0.006 


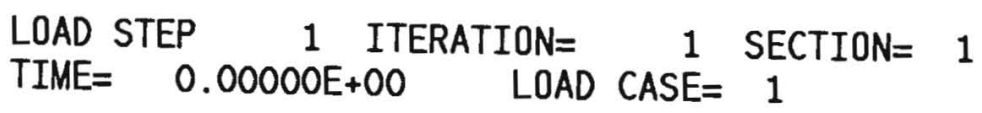

THE FOLLOWING $X, Y, Z$ DISPLACEMENTS ARE IN NODAL COORDINATES

\begin{tabular}{|c|c|c|c|c|c|c|}
\hline NODE & UX & UY & UZ & ROTX & ROTY & \\
\hline 337 & $0.00000000 E+00$ & $0.00000000 E+00$ & $0.69159279 \mathrm{E}-01$ & $0.34807504 \mathrm{E}-02$ & $-0.11420436 E-01$ & 0.00 \\
\hline 338 & $0.00000000 E+00$ & $0.00000000 E+00$ & 0.10176791 & & $-0.10434727 \mathrm{E}-01$ & \\
\hline 339 & $0.00000000 E+00$ & $0.00000000 E+00$ & 0.13234888 & & $0066686 \mathrm{E}-01$ & \\
\hline 340 & $0000000 E+00$ & $0.00000000 E+00$ & 0.16272243 & & $302594 \mathrm{E}-01$ & 008 \\
\hline 341 & $0000000 E+00$ & $0.00000000 E+00$ & 0.19441294 & & $843420 E-01$ & 00 \\
\hline 342 & $000000 E+00$ & $0.00000000 E+00$ & 0.19860100 & $2 \mathrm{E}-03$ & $6673 E-02$ & 0.000 \\
\hline 343 & $0000000 E+00$ & $0.00000000 E+00$ & 0.16964925 & $5 E-03$ & $5674 \mathrm{E}-02$ & 0.000 \\
\hline 344 & $0000000 E+00$ & $0.00000000 E+00$ & 0.14051392 & $0 E-03$ & 265417E-02 & 0.00 \\
\hline 345 & $0.00000000 E+00$ & $0.00000000 E+00$ & 0.11035216 & -03 & $0.10347433 E-01$ & 0.000 \\
\hline 346 & $0.00000000 E+00$ & $0.00000000 E+00$ & $0.77973014 \mathrm{E}-01$ & & $0.11324721 \mathrm{E}-01$ & 0.000 \\
\hline & $0.00000000 \mathrm{E}+00$ & $0000000 E+00$ & $0.41827254 \mathrm{E}-01$ & & $0.12878749 \mathrm{E}-01$ & \\
\hline & 00000 & $0000000 \mathrm{E}$ & $0.00000000 E+00$ & $0.73304887 E-03$ & $0.15133698 \mathrm{E}-01$ & \\
\hline
\end{tabular}

***** POST1 NODAL DISPLACEMENT LISTING *****

LOAD STEP 1 ITERATION $=1$ SECTION $=1$

TIME $=0.00000 \mathrm{E}+00 \quad$ LOAD CASE $=1$

THE FOLLOWING $X, Y, Z$ DISPLACEMENTS ARE IN NODAL COORDINATES

\begin{tabular}{lcccccc} 
NODE & $U X$ & $U Y$ & UZ & ROTX & \multicolumn{1}{l}{ ROTY } & F \\
349 & $0.00000000 E+00$ & $0.00000000 E+00$ & $-0.48784211 E-01$ & $0.75416064 \mathrm{E}-03$ & $0.17197891 \mathrm{E}-01$ & 0.006 \\
350 & $0.00000000 \mathrm{E}+00$ & $0.00000000 \mathrm{E}+00$ & -0.10210566 & $0.77840634 \mathrm{E}-03$ & $0.18179946 \mathrm{E}-01$ & 0.006 \\
351 & $0.00000000 \mathrm{E}+00$ & $0.00000000 \mathrm{E}+00-0.15690604$ & $0.80495789 \mathrm{E}-03$ & $0.18203913 \mathrm{E}-01$ & 0.006 \\
352 & $0.00000000 \mathrm{E}+00$ & $0.00000000 \mathrm{E}+00-0.21048531$ & $0.83283192 \mathrm{E}-03$ & $0.17383514 \mathrm{E}-01$ & 0.006 \\
353 & $0.00000000 \mathrm{E}+00$ & $0.00000000 \mathrm{E}+00$ & -0.26045142 & $0.86116330 \mathrm{E}-03$ & $0.15805152 \mathrm{E}-01$ & 0.006 \\
354 & $0.00000000 \mathrm{E}+00$ & $0.00000000 \mathrm{E}+00-0.30553641$ & $0.87117493 \mathrm{E}-03$ & $0.14159815 \mathrm{E}-01$ & 0.006 \\
355 & $0.00000000 \mathrm{E}+00$ & $0.00000000 \mathrm{E}+00-0.34488359$ & $0.88057278 \mathrm{E}-03$ & $0.11989743 \mathrm{E}-01$ & 0.006 \\
356 & $0.00000000 \mathrm{E}+00$ & $0.00000000 \mathrm{E}+00-0.37703429$ & $0.88877319 \mathrm{E}-03$ & $0.93792268 \mathrm{E}-02$ & 0.006 \\
357 & $0.00000000 \mathrm{E}+00$ & $0.00000000 \mathrm{E}+00-0.40082672$ & $0.89516684 \mathrm{E}-03$ & $0.64362949 \mathrm{E}-02$ & 0.006 \\
$\Rightarrow 358$ & $0.00000000 \mathrm{E}+00$ & $0.00000000 \mathrm{E}+00-0.41543180-$ & $0.89923272 \mathrm{E}-03$ & $0.32729595 \mathrm{E}-02$ & 0.006 \\
359 & $0.00000000 \mathrm{E}+00$ & $0.00000000 \mathrm{E}+00-0.42035492-$ & $0.90062771 \mathrm{E}-03$ & $-0.23084177 \mathrm{E}-09$ & 0.006 \\
360 & $0.00000000 \mathrm{E}+00$ & $0.00000000 \mathrm{E}+00-0.41543180-$ & $0.89923269 \mathrm{E}-03$ & $-0.32729599 \mathrm{E}-02$ & 0.006
\end{tabular}

***** POST1 NODAL DISPLACEMENT LISTING *****

LOAD STEP 1 ITERATION $=1$ SECTION $=1$

TIME $=0.00000 \mathrm{E}+00 \quad$ LOAD CASE $=1$

THE FOLLOWING $X, Y, Z$ DISPLACEMENTS ARE IN NODAL COORDINATES

$\begin{array}{lcccccc}\text { NODE } & \text { UX } & \text { UY } & \text { UZ } & \text { ROTX } & \text { ROTY } & F \\ 361 & 0.00000000 E+00 & 0.00000000 E+00 & -0.40082672 & 0.89516677 E-03 & -0.64362953 E-02 & 0.006 \\ 362 & 0.00000000 E+00 & 0.00000000 E+00 & -0.37703428 & 0.88877309 E-03 & -0.93792272 E-02 & 0.006 \\ 363 & 0.00000000 E+00 & 0.00000000 E+00 & -0.34488358 & 0.88057265 E-03 & -0.11989744 E-01 & 0.006 \\ 364 & 0.00000000 E+00 & 0.00000000 E+00 & -0.30553641 & 0.87117476 E-03 & -0.14159815 E-01 & 0.006 \\ 365 & 0.00000000 E+00 & 0.00000000 E+00 & -0.26045141 & 0.86116309 E-03 & -0.15805152 E-01 & 0.006 \\ 366 & 0.00000000 E+00 & 0.00000000 E+00 & -0.21048531 & 0.83283171 E-03 & -0.17383514 E-01 & 0.006 \\ 367 & 0.00000000 E+00 & 0.00000000 E+00 & -0.15690604 & 0.80495768 E-03 & -0.18203913 E-01 & 0.006 \\ 368 & 0.00000000 E+00 & 0.00000000 E+00 & -0.10210566 & 0.77840613 E-03 & -0.18179946 E-01 & 0.006 \\ 369 & 0.00000000 E+00 & 0.00000000 E+00 & -0.48784209 E-01 & 0.75416043 E-03 & -0.17197891 E-01 & 0.006 \\ 370 & 0.00000000 E+00 & 0.00000000 E+00 & 0.00000000 E+00 & 0.73304866 E-03 & -0.15133697 E-01 & 0.006 \\ 371 & 0.00000000 E+00 & 0.00000000 E+00 & 0.41827251 E-01 & 0.71551821 E-03 & -0.12878749 E-01 & 0.006\end{array}$


***** POST1 NODAL DISPLACEMENT LISTING *****

LOAD STEP 1 ITERATION $=1$ SECTION $=1$

TIME $=0.00000 \mathrm{E}+00 \quad$ LOAD CASE $=1$

THE FOLLOWING $X, Y, Z$ DISPLACEMENTS ARE IN NODAL COORDINATES

\begin{tabular}{|c|c|c|c|c|c|c|}
\hline NODE & UX & UY & UZ & ROTX & ROTY & \\
\hline 373 & $0.00000000 E+00$ & $0.00000000 E+00$ & 0.11035215 & $3065051 \mathrm{E}$ & $-0.10347432 E-01$ & \\
\hline & $0.00000000 \mathrm{E}+00$ & $0.00000000 E+00$ & 0.14051391 & $8214850 \mathrm{E}-03$ & $-0.98265410 \mathrm{E}-02$ & 0.006 \\
\hline 375 & $0.00000000 E+00$ & $0.00000000 E+00$ & 0.16964923 & $0.67524096 \mathrm{E}-03$ & $-0.96435666 \mathrm{E}-02$ & 0.000 \\
\hline 376 & $0.00000000 E+00$ & $0.00000000 E+00$ & 0.19860098 & $0.66920164 \mathrm{E}-03$ & $-0.96866664 \mathrm{E}-02$ & \\
\hline 377 & $0.00000000 E+00$ & $0.00000000 \mathrm{E}+00$ & 0.19815154 & $-0.30450532 \mathrm{E}-03$ & $0.10949587 \mathrm{E}-01$ & 0.006 \\
\hline 378 & $0.00000000 E+00$ & $0.00000000 \mathrm{E}+00$ & 0.16606493 & $-0.16470904 E-02$ & $0.10465583 \mathrm{E}-01$ & $0.00 c$ \\
\hline 379 & $0.00000000 \mathrm{E}+00$ & $0.00000000 E+00$ & 0.13517013 & $-0.25372271 \mathrm{E}-02$ & $0.10258323 \mathrm{E}-01$ & 0.006 \\
\hline 380 & $0.00000000 E+00$ & $0.00000000 \mathrm{E}+00$ & 0.10403331 & $-0.30888688 \mathrm{E}-02$ & $0.10621259 \mathrm{E}-01$ & 0.006 \\
\hline 381 & $0.00000000 E+00$ & $0.00000000 E+00$ & $0.70945337 \mathrm{E}-01$ & $-0.34950273 \mathrm{E}-02$ & $0.11558312 \mathrm{E}-01$ & \\
\hline 382 & $0.00000000 E+00$ & & $0.34172263 \mathrm{E}-01$ & $-0.38065671 \mathrm{E}-02$ & $0.13078445 E-01$ & 0.006 \\
\hline 383 & $0.00000000 \mathrm{E}+00$ & $0.00000000 E+00$ & $-0.79433368 \mathrm{E}-02$ & $-0.38938884 E-02$ & $0.15048206 \mathrm{E}-01$ & 0.0 \\
\hline 384 & $0.00000000 E+00$ & $0.00000000 \mathrm{E}+00$ & $-0.55913188 \mathrm{E}-$ & $-0.35183754 E-02$ & $0.16790921 \mathrm{E}-01$ & 0.06 \\
\hline
\end{tabular}

***** POST1 NODAL DISPLACEMENT LISTING *****

LOAD STEP 1 ITERATION= 1 SECTION $=1$

TIME $=0.00000 \mathrm{E}+00 \quad$ LOAD CASE $=1$

THE FOLLOWING $X, Y, Z$ DISPLACEMENTS ARE IN NODAL COORDINATES

$\begin{array}{lccc}\text { NODE } & U X & U Y & U Z \\ 385 & 0.00000000 E+00 & 0.00000000 E+00 & -0.10782528 \\ 386 & 0.00000000 E+00 & 0.00000000 E+00 & -0.16042184 \\ 387 & 0.00000000 E+00 & 0.00000000 E+00 & -0.21047467 \\ 388 & 0.00000000 E+00 & 0.00000000 E+00 & -0.25725318 \\ 389 & 0.00000000 E+00 & 0.00000000 E+00 & -0.30450122 \\ 390 & 0.00000000 E+00 & 0.00000000 E+00 & -0.34606057 \\ 391 & 0.00000000 E+00 & 0.00000000 E+00 & -0.37891059 \\ 392 & 0.00000000 E+00 & 0.00000000 E+00 & -0.40263154 \\ 393 & 0.00000000 E+00 & 0.00000000 E+00 & -0.41701699 \\ 394 & 0.00000000 E+00 & 0.00000000 E+00 & -0.42184423 \\ 395 & 0.00000000 E+00 & 0.00000000 E+00 & -0.41701699 \\ 396 & 0.00000000 E+00 & 0.00000000 E+00 & -0.40263154\end{array}$

ROTX
$-0.28000902 \mathrm{E}-02$
$-0.16946383 \mathrm{E}-02$
$-0.14623594 \mathrm{E}-03$
$0.12808749 \mathrm{E}-02$
$0.43183944 \mathrm{E}-03$
$-0.35982651 \mathrm{E}-03$
$-0.63363728 \mathrm{E}-03$
$-0.58074496 \mathrm{E}-03$
$-0.46257226 \mathrm{E}-03$
$-0.41122429 \mathrm{E}-03$
$-0.46257229 \mathrm{E}-03$
$-0.58074502 \mathrm{E}-03$
ROTY

$0.17586865 \mathrm{E}-01$

$0.17230950 \mathrm{E}-01$

$0.15995223 \mathrm{E}-01$

$0.15610049 \mathrm{E}-01$

$0.15139524 \mathrm{E}-01$

$0.12455528 \mathrm{E}-01$

$0.94349893 \mathrm{E}-02$

$0.63591759 \mathrm{E}-02$

$0.32091696 \mathrm{E}-02$

$-0.11577569 \mathrm{E}-09$

$-0.32091698 \mathrm{E}-02$

$-0.63591761 E-02$
0.006
0.006
0.006
0.000
0.006
$0.00 C$
0.006
$0.00 C$
0.006
0.006
0.006
$0.00 C$

\section{***** POST1 NODAL DISPLACEMENT LISTING *****}

LOAD STEP 1 ITERATION $=1$ SECTION $=1$
TIME= $0.00000 E+\infty$ LOAD CASE $=1$

THE FOLLOWING $X, Y, Z$ DISPLACEMENTS ARE IN NODAL COORDINATES
NODE
397
ux
$0.00000000 E+\infty 0$
UY
UZ
$398 \quad 0.00000000 E+00$
$399 \quad 0.00000000 E+00$
$400 \quad 0.00000000 E+00$
$401 \quad 0.00000000 E+00$
$402 \quad 0.00000000 E+00$
403
$0.00000000 E+00-0.37891059$
$0.00000000 E+00-0.34606057$
$0.00000000 E+00-0.30450122$
$0.00000000 E+00-0.25725318$
$0.00000000 E+00-0.21047467$
$0.00000000 E+00-0.16042184$
$0.00000000 E+\infty 0-0.10782528$

ROTX $-0.63363737 \mathrm{E}-03-0.94349895 \mathrm{E}-02$ $-0.35982663 \mathrm{E}-03-0.12455528 \mathrm{E}-01$

$0.43183923 \mathrm{E}-03-0.15139524 \mathrm{E}-01$

$0.12808746 \mathrm{E}-02-0.15610049 \mathrm{E}-01$

$-0.14623640 \mathrm{E}-03-0.15995223 \mathrm{E}-01$

$-0.16946389 \mathrm{E}-02-0.17230950 \mathrm{E}-01$

$-0.28000909 \mathrm{E}-02-0.17586865 \mathrm{E}-01$
0.006

0.006

0.006

$0.00 \mathrm{C}$

0.006

0.006

0.006 
404

405

406

407

408
$0.00000000 E+00$

$0.00000000 E+00$

$0.00000000 E+00$

$0.00000000 E+00$

$0.00000000 E+00$
$0.00000000 E+00$

$0.00000000 E+00$

$-0.55913190 \mathrm{E}-01-0.35183763 \mathrm{E}-02$

$0.00000000 E+00$

$0.00000000 E+00$

$0.00000000 E+00$
$-0.79433415 \mathrm{E}-02$

$0.34172258 \mathrm{E}-01$

$0.70945329 \mathrm{E}-01$

0.10403330
$-0.38938893 \mathrm{E}-02$

$-0.16790921 \mathrm{E}-01$

$-0.15048205 \mathrm{E}-01$

$-0.38065680 \mathrm{E}-02-0.13078445 \mathrm{E}-01$

$-0.34950281 \mathrm{E}-02$

$-0.30888695 \mathrm{E}-02$

$-0.11558311 \mathrm{E}-01$

***** POST1 NODAL DISPLACEMENT LISTING *****

LOAD STEP 1 ITERATION $=1$ SECTION $=1$

TIME $=0.00000 \mathrm{E}+00 \quad$ LOAD CASE $=1$

THE FOLLOWING $X, Y, Z$ DISPLACEMENTS ARE IN NODAL COORDINATES

\begin{tabular}{|c|c|c|c|c|c|c|}
\hline NODE & UX & UY & UZ & ROTX & ROTY & \\
\hline 409 & $0.00000000 E+00$ & $0.00000000 E+00$ & 0.13517012 & $-0.25372279 \mathrm{E}-02$ & $-0.10258322 \mathrm{E}-01$ & 0.006 \\
\hline 410 & $0.00000000 E+00$ & $0.00000000 E+00$ & 0.16606491 & $-0.16470914 \mathrm{E}-02$ & $-0.10465582 \mathrm{E}-01$ & 0.006 \\
\hline 411 & $0.00000000 E+00$ & $0.00000000 \mathrm{E}+00$ & 0.19815151 & $-0.30450645 E-03$ & $-0.10949586 \mathrm{E}-01$ & 0.006 \\
\hline 412 & $0.00000000 E+00$ & $0.00000000 E+00$ & 0.19914771 & $0.10980162 \mathrm{E}-02$ & $0.11928876 \mathrm{E}-01$ & $.00 c$ \\
\hline 413 & $0.00000000 E+00$ & $0.00000000 E+00$ & 0.16411797 & $0.78075533 \mathrm{E}-03$ & $0.11292795 \mathrm{E}-01$ & 0.000 \\
\hline 414 & $0.00000000 E+00$ & $0.00000000 E+00$ & 0.13104066 & $0.38034239 \mathrm{E}-03$ & $0.10861354 \mathrm{E}-01$ & 0 \\
\hline 415 & $0.00000000 E+00$ & $0.00000000 E+00$ & $0.98287188 \mathrm{E}-01$ & $-0.52891427 E-04$ & $0.11093087 \mathrm{E}-01$ & \\
\hline 416 & $0.00000000 E+00$ & $0.00000000 E+00$ & $0.63908666 \mathrm{E}-01$ & $-0.44584613 \mathrm{E}-03$ & $0.11927596 \mathrm{E}-01$ & \\
\hline 417 & $0.00000000 E+00$ & $0.00000000 E+00$ & $0.26225207 \mathrm{E}-01$ & $39826472 E-03$ & $0.13270484 \mathrm{E}-01$ & 0.0 \\
\hline 418 & $0.00000000 E+00$ & $0.00000000 E+00$ & $-0.16016817 \mathrm{E}-01$ & $9810526 \mathrm{E}$ & $0.14895790 \mathrm{E}-01$ & \\
\hline $\begin{array}{l}419 \\
420\end{array}$ & $\begin{array}{l}0.00000000 E+00 \\
0.00000000 E+00\end{array}$ & $0.00000000 \mathrm{E}+00$ & $-0.62916155 \mathrm{E}-01$ & $-0.39210202 E-$ & $0.16269260 \mathrm{E}-01$ & \\
\hline & & $.00000000 t+\infty$ & -0.11279563 & 0.13733273 & 0.16793089 & \\
\hline
\end{tabular}

***** POST1 NODAL DISPLACEMENT LISTING *****

LOAD STEP 1 ITERATION $=1$ SECTION $=1$

TIME $=0.00000 \mathrm{E}+00 \quad$ LOAD CASE $=1$

THE FOLLOWING $X, Y, Z$ DISPLACEMENTS ARE IN NODAL COORDINATES

$\begin{array}{lccc}\text { NODE } & U X & U Y & U Z \\ 421 & 0.00000000 E+00 & 0.00000000 E+00 & -0.16252839 \\ 422 & 0.00000000 E+00 & 0.00000000 E+00 & -0.20890876 \\ 423 & 0.00000000 E+00 & 0.0000000 E+00 & -0.25272911 \\ 424 & 0.00000000 E+00 & 0.00000000 E+00 & -0.30088922 \\ 425 & 0.00000000 E+00 & 0.00000000 E+00 & -0.34334570 \\ 426 & 0.00000000 E+00 & 0.00000000 E+00 & -0.37614058 \\ 427 & 0.00000000 E+00 & 0.00000000 E+00 & -0.39931866 \\ 428 & 0.00000000 E+00 & 0.00000000 E+00 & -0.41319585 \\ 429 & 0.00000000 E+00 & 0.00000000 E+00 & -0.41782748 \\ 430 & 0.00000000 E+00 & 0.00000000 E+00 & -0.41319585 \\ 431 & 0.00000000 E+00 & 0.00000000 E+00 & -0.39931866 \\ 432 & 0.00000000 E+00 & 0.00000000 E+00 & -0.37614058\end{array}$

ROTX
$0.75215000 \mathrm{E}-03$
$0.13236588 \mathrm{E}-02$
$0.17351342 \mathrm{E}-02$
$0.20703569 \mathrm{E}-02$
$0.24791072 \mathrm{E}-02$
$0.28760013 \mathrm{E}-02$
$0.32012762 \mathrm{E}-02$
$0.34136727 \mathrm{E}-02$
$0.34873702 \mathrm{E}-02$
$0.34136727 \mathrm{E}-02$
$0.32012761 \mathrm{E}-02$
$0.28760012 \mathrm{E}-02$

ROTY

$0.16163622 \mathrm{E}-01$

$0.14788337 \mathrm{E}-01$

$0.15413760 \mathrm{E}-01$

$0.15514458 \mathrm{E}-01$

$0.12566508 \mathrm{E}-01$

$0.93038991 \mathrm{E}-02$

$0.61684323 \mathrm{E}-02$

$0.30867094 \mathrm{E}-02$

$0.12750216 \mathrm{E}-09$

$-0.30867092 \mathrm{E}-02$

(61684320E-02

***** POST1 NODAL DISPLACEMENT LISTING *****

\section{LOAD STEP 1 ITERATION= 1 SECTION= 1}

TIME $=0.00000 \mathrm{E}+00 \quad$ LOAD CASE $=1$

THE FOLLOWING $X, Y, Z$ DISPLACEMENTS ARE IN NODAL COORDINATES

NODE

UX

UY

UZ

ROTX

ROTY

$433 \quad 0.00000000 E+00$

$0.00000000 E+00-0.34334570$

434

$0.00000000 E+00$

$0.00000000 E+00-0.30088922$

435

$0.00000000 E+00$

$0.00000000 \mathrm{E}+00-0.25272912$

$0.24791070 \mathrm{E}-02-0.12566507 \mathrm{E}-01$

$0.20703567 \mathrm{E}-02-0.15514458 \mathrm{E}-01$

$0.17351338 \mathrm{E}-02-0.15413760 \mathrm{E}-01$

0.006
0.006
0.006
0.006
0.006 
$\begin{array}{ll}436 & 0.00000000 E+00 \\ 437 & 0.00000000 E+00 \\ 438 & 0.00000000 E+00 \\ 439 & 0.00000000 E+00 \\ 440 & 0.00000000 E+00 \\ 441 & 0.00000000 E+00 \\ 442 & 0.00000000 E+00 \\ 443 & 0.00000000 E+00 \\ 444 & 0.00000000 E+00\end{array}$

$0.00000000 E+00-0.20890876$

$0.00000000 E+00-0.16252840$

$0.00000000 E+00-0.11279563$

$0.00000000 \mathrm{E}+00$

$-0.62916162 \mathrm{E}-0$

$0.00000000 \mathrm{E}+00$

$-0.16016828 \mathrm{E}-01$

$0.00000000 \mathrm{E}+00$

$0.26225196 \mathrm{E}-01$

$0.63908653 \mathrm{E}-01$

$0.98287174 \mathrm{E}-01$

0.13104064

0.0000

***** POST1 NODAL DISPLACEMENT LISTING

LOAD STEP 1 ITERATION $=1$ SECTION $=1$

TIME $=0.00000 E+00 \quad$ LOAD CASE $=1$

THE FOLLOWING $X, Y, Z$ DISPLACEMENTS ARE IN NODAL COORDINATES

$\begin{array}{lccc}\text { NODE } & U X & U Y & U Z \\ 445 & 0.00000000 E+00 & 0.00000000 E+00 & 0.16411794 \\ 446 & 0.00000000 E+00 & 0.00000000 E+00 & 0.19914768 \\ 447 & 0.00000000 E+00 & 0.00000000 E+00 & 0.20489207 \\ 448 & 0.00000000 E+00 & 0.00000000 E+00 & 0.17106578 \\ 449 & 0.00000000 E+00 & 0.00000000 E+00 & 0.13796763 \\ 450 & 0.00000000 E+00 & 0.00000000 E+00 & 0.10442626 \\ 451 & 0.00000000 E+00 & 0.00000000 E+00 & 0.69139358 E-01 \\ 452 & 0.00000000 E+00 & 0.00000000 E+00 & 0.30930459 E-01 \\ 453 & 0.00000000 E+00 & 0.00000000 E+00 & -0.11200439 E-01 \\ 454 & 0.00000000 E+00 & 0.00000000 E+00 & -0.57428080 E-01 \\ 455 & 0.00000000 E+00 & 0.00000000 E+00 & -0.10634125 \\ 456 & 0.00000000 E+00 & 0.00000000 E+00 & -0.15549281\end{array}$

$0.13236583 \mathrm{E}-02-0.14788337 \mathrm{E}-01$

$0.75214932 \mathrm{E}-03-0.16163621 \mathrm{E}-01$

$0.13733185 \mathrm{E}-03-0.16793089 \mathrm{E}-01$

$-0.39210306 \mathrm{E}-03-0.16269260 \mathrm{E}-01$

$-0.69810637 \mathrm{E}-03-0.14895790 \mathrm{E}-01$

$-0.69826582 \mathrm{E}-03-0.13270484 \mathrm{E}-01$

$-0.44584717 \mathrm{E}-03-0.11927596 \mathrm{E}-01$

$-0.52892347 \mathrm{E}-04-0.11093087 \mathrm{E}-01$

$0.38034158 \mathrm{E}-03-0.10861354 \mathrm{E}-01$
0.006

0.006

0.006

0.006

0.006

0.006

0.006

0.006

0.006

\section{***** POST1 NODAL DISPLACEMENT LISTING *****}

LOAD STEP 1 ITERATION $=1$ SECTION $=1$

TIME $=0.00000 \mathrm{E}+00 \quad$ LOAD CASE $=1$

$\begin{array}{ccc}\text { ROTX } & \text { ROTY } & \text { F } \\ 0.78075462 \mathrm{E}-03 & -0.11292794 \mathrm{E}-01 & 0.00 \mathrm{C} \\ 0.10980156 \mathrm{E}-02 & -0.11928876 \mathrm{E}-01 & 0.006 \\ 0.26279438 \mathrm{E}-02 & 0.11420689 \mathrm{E}-01 & 0.006 \\ 0.35279023 \mathrm{E}-02 & 0.11121873 \mathrm{E}-01 & 0.00 \mathrm{C} \\ 0.38327898 \mathrm{E}-02 & 0.11029295 \mathrm{E}-01 & 0.006 \\ 0.37355212 \mathrm{E}-02 & 0.11398770 \mathrm{E}-01 & 0.006 \\ 0.35414331 \mathrm{E}-02 & 0.12183726 \mathrm{E}-01 & 0.006 \\ 0.34507574 \mathrm{E}-02 & 0.13346266 \mathrm{E}-01 & 0.006 \\ 0.35219181 \mathrm{E}-02 & 0.14760088 \mathrm{E}-01 & 0.006 \\ 0.36542200 \mathrm{E}-02 & 0.15973911 \mathrm{E}-01 & 0.006 \\ 0.37842930 \mathrm{E}-02 & 0.16491993 \mathrm{E}-01 & 0.006 \\ 0.36324890 \mathrm{E}-02 & 0.16108976 \mathrm{E}-01 & 0.006\end{array}$

THE FOLLOWING $X, Y, Z$ DISPLACEMENTS ARE IN NODAL COORDINATES

$\begin{array}{cccc}\text { NODE } & U X & U Y & U Z \\ 457 & 0.00000000 E+00 & 0.00000000 E+00 & -0.20234551 \\ 458 & 0.00000000 E+00 & 0.00000000 E+00 & -0.24689055 \\ 459 & 0.00000000 E+00 & 0.00000000 E+00 & -0.29228003 \\ 460 & 0.00000000 E+00 & 0.00000000 E+00 & -0.33153878 \\ 461 & 0.00000000 E+00 & 0.00000000 E+00 & -0.36216951 \\ 462 & 0.00000000 E+00 & 0.00000000 E+00 & -0.38406855 \\ 463 & 0.00000000 E+00 & 0.00000000 E+00 & -0.39726451 \\ 464 & 0.00000000+00 & 0.00000000 E+00 & -0.40167919 \\ 465 & 0.00000000 E+00 & 0.00000000 E+00 & -0.39726452 \\ 466 & 0.00000000 E+00 & 0.00000000 E+00 & -0.38406855 \\ 467 & 0.00000000 E+00 & 0.00000000 E+00 & -0.36216952 \\ 468 & 0.00000000 E+00 & 0.00000000 E+00 & -0.33153879\end{array}$

ROTX
$0.29437155 \mathrm{E}-02$
$0.21411589 \mathrm{E}-02$
$0.35237680 \mathrm{E}-02$
$0.49434759 \mathrm{E}-02$
$0.58330054 \mathrm{E}-02$
$0.62875911 \mathrm{E}-02$
$0.65005155 \mathrm{E}-02$
$0.65639014 \mathrm{E}-02$
$0.65005155 \mathrm{E}-02$
$0.62875911 \mathrm{E}-02$
$0.58330054 \mathrm{E}-02$
$0.49434759 \mathrm{E}-02$

$0.15055787 \mathrm{E}-01$

$0.15217476 \mathrm{E}-01$

$0.14418014 \mathrm{E}-01$

$0.11694489 E-01$

$0.87577306 \mathrm{E}-02$

$0.58552827 \mathrm{E}-02$

$0.29413845 \mathrm{E}-02$

$0.30460858 \mathrm{E}-09$

$-0.29413840 E-02$

$-0.58552822 \mathrm{E}-02$

$-0.87577301 E-02$

$-0.11694489 \mathrm{E}-01$
0.00

0.006

0.006

0.006

0.006

0.006

0.006

$0.00 C$

$0.00 C$

$0.00 C$

0.006

$0.00 C$

***** POST1 NODAL DISPLACEMENT LISTING *****

LOAD STEP 1 ITERATION= 1 SECTION $=1$

TIME $=0.00000 \mathrm{E}+\infty \quad$ LOAD CASE $=1$

THE FOLLOWING $X, Y, Z$ DISPLACEMENTS ARE IN NODAL COORDINATES 


$\begin{array}{lcccccc}\text { NODE } & U X & U Y & U Z & \text { ROTX } & \text { ROTY } & F \\ 469 & 0.00000000 E+00 & 0.00000000 E+00 & -0.29228004 & 0.35237680 E-02 & -0.14418014 E-01 & 0.006 \\ 470 & 0.00000000 E+00 & 0.00000000 E+00 & -0.24689056 & 0.21411586 E-02 & -0.15217476 E-01 & 0.006 \\ 471 & 0.00000000 E+00 & 0.00000000 E+00 & -0.20234552 & 0.29437150 E-02 & -0.15055787 E-01 & 0.006 \\ 472 & 0.00000000 E+00 & 0.00000000 E+00 & -0.15549283 & 0.36324883 E-02 & -0.16108975 E-01 & 0.006 \\ 473 & 0.00000000 E+00 & 0.00000000 E+00 & -0.10634127 & 0.37842922 E-02 & -0.16491992 E-01 & 0.006 \\ 474 & 0.00000000 E+00 & 0.00000000 E+00 & -0.57428097 E-01 & 0.36542191 E-02 & -0.15973911 E-01 & 0.006 \\ 475 & 0.00000000 E+00 & 0.00000000 E+00 & -0.11200456 E-01 & 0.35219172 E-02 & -0.14760088 E-01 & 0.006 \\ 476 & 0.00000000 E+00 & 0.00000000 E+00 & 0.30930440 E-01 & 0.34507565 E-02 & -0.13346266 E-01 & 0.006 \\ 477 & 0.00000000 E+00 & 0.00000000 E+00 & 0.69139340 E-01 & 0.35414322 E-02 & -0.12183726 E-01 & 0.006 \\ 478 & 0.00000000 E+00 & 0.00000000 E+00 & 0.10442625 & 0.37355204 E-02 & -0.11398770 E-01 & 0.006 \\ 479 & 0.00000000 E+00 & 0.00000000 E+00 & 0.13796761 & 0.38327892 E-02 & -0.11029295 E-01 & 0.006 \\ 480 & 0.00000000 E+00 & 0.00000000 E+00 & 0.17106575 & 0.35279021 E-02 & -0.11121873 E-01 & 0.006\end{array}$

\section{POST1 NODAL DISPLACEMENT LISTING}

LOAD STEP 1 ITERATION $=1$ SECTION $=1$

TIME $=0.00000 \mathrm{E}+00 \quad$ LOAD CASE $=1$

THE FOLLOWING $X, Y, Z$ DISPLACEMENTS ARE IN NODAL COORDINATES

$\begin{array}{lcccccc}\text { NODE } & \text { UX } & \text { UY } & \text { UZ } & \text { ROTX } & \text { ROTY } & \text { F } \\ 481 & 0.00000000 E+00 & 0.00000000 E+00 & 0.20489204 & 0.26279440 E-02 & -0.11420688 E-01 & 0.006\end{array}$ MAXIMUMS

$\begin{array}{llll}\text { NODE } & 0 & 0 & 289\end{array}$

VALUE $\quad 0.00000000 E+00 \quad 0.00000000 E+00 \quad-0.43550697$

ROUTINE COMPLETED $* * * * * \quad \mathrm{CP}=\quad 5.710$

ENTER HELP FOR LIST OF OPTIONS

ENTER /EOF TO EXIT

ANSYS(R) COPYRIGHT(C) 1971, 1978, 1982, 1983, 1985, 1987

SWANSON ANALYSIS SYSTEMS, INC. AS AN UNPUBLISHED WORK.

PROPRIETARY DATA - UNAUTHORIZED USE, DISTRIBUTION, OR DUPLICATION

IS PROHIBITED. ALL RIGHTS RESERVED.

SYSTEM $=$ (FERMILAB) UPDATE $=350$ A 21

FOR SUPPORT CALL MARK LEININGER PHONE (312) 840-4776 TWX

CURRENT JOBNAME=FILE

/EOF ENCOUNTERED ON FILE 5

DATA INPUT WRITTEN ON FILE18

***** RUN COMPLETED ***** CP= $\quad 5.7600$ TIME $=\quad 9.4998$ 
BEAMAC II beam name: platform3b $\frac{N 1}{0.00 e+0} \frac{2.17 e+1}{1} \frac{2.17 e+1}{1}$ D1Y $1.50 \mathrm{e}+1$

Load

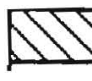

APPENDIX C

Last modified at 3:09:36 PM on Wed, Dec 28, 1988

$\frac{{ }^{E 2}}{6.36 \mathrm{e}+1}$

$\frac{\mathrm{N3}}{8.53 \mathrm{e}+1} \frac{\mathrm{E3}}{1} \frac{\mathrm{E3} \mathrm{e}+1}{1} \frac{1.07 \mathrm{~N} e+2}{1}$ $1.50 \theta+1$

Tension
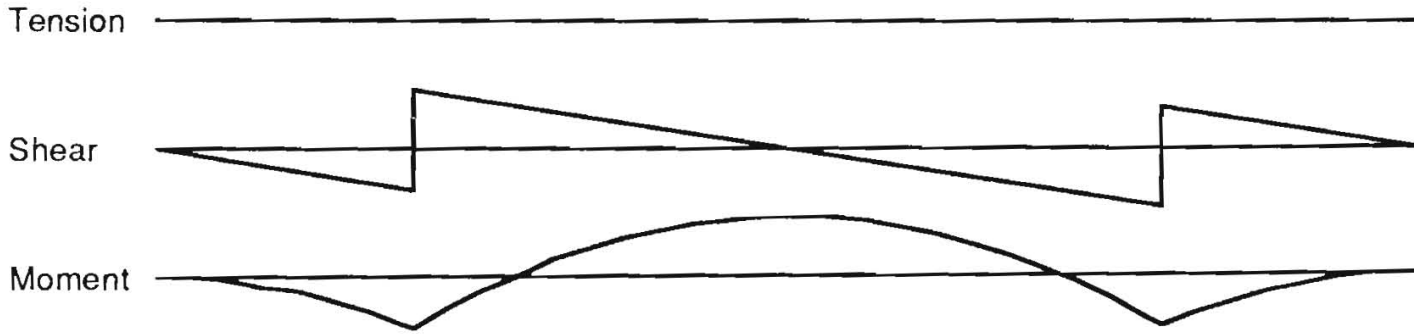

Defl.

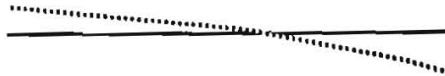

60

$F:$ lb

$D: I b /$ in

M: Ib-in

$0.00 e+0$

$\mathrm{lb}$

$0.00 e+0$

$4.77 \mathrm{e}+2$

$\mathrm{lb}$

$-4.77 e+2$

$4.04 e+3$

Ib-in

$-3.54 e+3$

$1.35 e-1$

inch

$-3.01 \mathrm{e}-1$

Distance $0.00 \mathrm{e}+0$

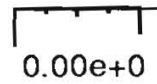

\begin{abstract}
$2.14 \mathrm{e}+1$
\end{abstract}
$L /\left.3\right|_{4.28 e+1} ^{L L / 2}$

$2 \mathrm{~L} / 3$
$6.42 \mathrm{e}+1$

$8.56 \mathrm{e}+1$

inch

$1.07 e+2$ 
Input Data

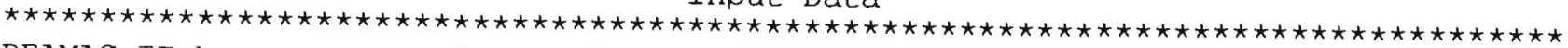

BEAMAC II beam name: platform3b.

Last modified at 3:09:36 PM on Wed, Dec 28, 1988.

All distances are given in inch from the left end of the beam.

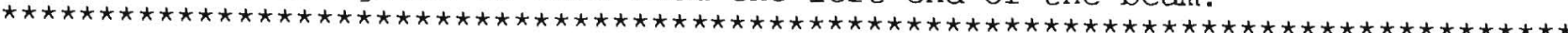

Beam length is 1.07 e+2 inch.

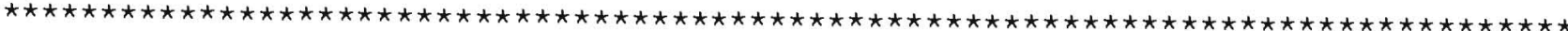
Node Location Hinge

No. inch

\begin{tabular}{|c|c|c|c|c|c|}
\hline 1 & $0.00 e+0$ & none & No & & \\
\hline 2 & $2.17 e+1$ & pin & No & & \\
\hline 3 & $8.53 e+1$ & roller & No & & \\
\hline 4 & $1.07 e+2$ & none & No & & \\
\hline 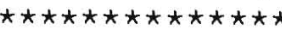 & 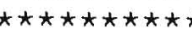 & 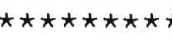 & $\star \star \star \star \star \star \star \star \star ~$ & $\star \star \star \star \star \star \star \star \star \star ~$ & 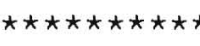 \\
\hline Element & From, To & Length & Section & $E$ & \\
\hline No. & Nodes & inch & name & psi & \\
\hline 1 & 1,2 & $2.17 e+1$ & --- & $1.00 \mathrm{e}+7$ & \\
\hline 2 & 2,3 & $6.36 \mathrm{e}+1$ & --- & $1.00 e+7$ & \\
\hline 3 & 3,4 & $2.17 e+1$ & . $\quad---$ & $1.00 e+7$ & \\
\hline 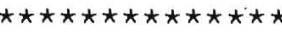 & 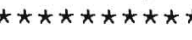 & 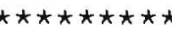 & 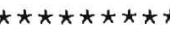 & 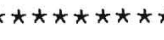 & 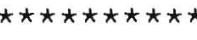 \\
\hline Element & Area & Depth & Iz & Weight & Include \\
\hline No. & $\operatorname{in} \star \star 2$ & in & $\operatorname{in} \star \star 4$ & 1b/in & self wt. \\
\hline 1 & 4.31 & 16.00 & 0.47 & 0.42 & No \\
\hline 2 & 4.31 & 16.00 & 0.47 & 0.42 & No \\
\hline 3 & 4.31 & 16.00 & 0.47 & 0.42 & No \\
\hline
\end{tabular}

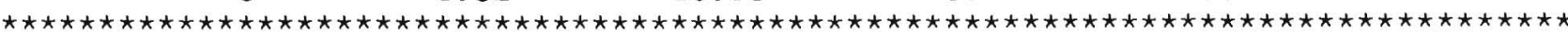

Concentrated Forces:

(none)

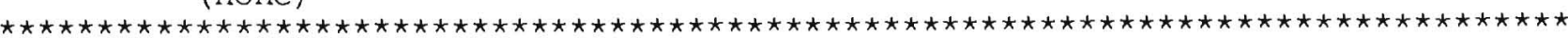

Distributed Loads:

No. $L$ end at $R$ end at MagX at $L$ MagY at $L$ MagX at $R$ MagY at $R$

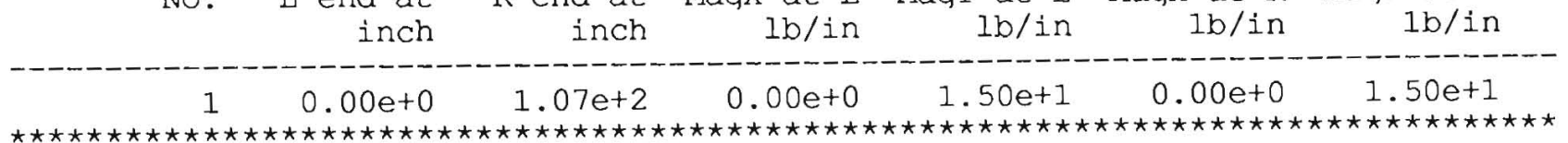

Applied Moments:

(none)

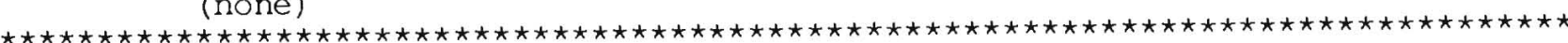




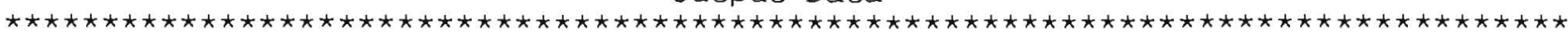

BEAMAC II beam name: platform 3 b.

Last modified at 3:09:36 PM on Wed, Dec 28, 1988.

All distances are given in inch from the left end of the beam.

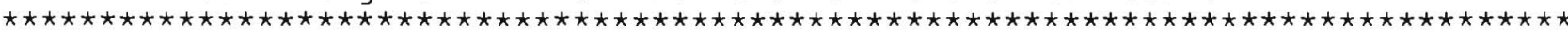

Maximum tension is $0.00 \mathrm{e}+0 \mathrm{lb}$; it occurs:

from $\mathrm{x}=0.00 \mathrm{e}+0$ inch to $\mathrm{x}=1.07 \mathrm{e}+2$ inch.

Maximum compression is $-0.00 \mathrm{e}+0$ 1b; it occurs:

from $x=0.00 e+0$ inch to $x=1.07 e+2$ inch.

Maximum shear is $4.77 \mathrm{e}+2 \mathrm{lb}$; it occurs: at $\mathrm{x}=2.17 \mathrm{e}+1$ inch.

Minimum shear is $-4.77 e+2$ 1b; it occurs: at $x=8.53 e+1$ inch.

Maximum moment is $4.04 \mathrm{e}+3 \mathrm{lb}-\mathrm{in}$; it occurs: at $x=5.35 \mathrm{e}+1$ inch.

Minimum moment is $-3.54 \mathrm{e}+3 \mathrm{lb}-\mathrm{in}$; it occurs:

at $x=2.17 \mathrm{e}+1$ inch;

at $x=8.53 e+1$ inch.

The maximum upward deflection is approximately $1.35 e-1$ inch.

The maximum downward deflection is approximately $3.01 \mathrm{e}-1$ inch.

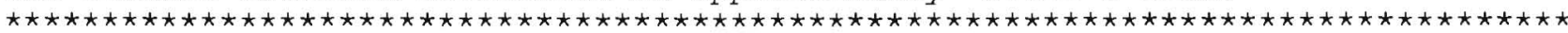

Node deformations: (positive is to right, up, or counterclockwise.)

\begin{tabular}{ccccc}
$\begin{array}{c}\text { Node } \\
\text { No. }\end{array}$ & $\begin{array}{c}\text { Location } \\
\text { inch }\end{array}$ & $\begin{array}{c}\text { Delta-X } \\
\text { inch }\end{array}$ & $\begin{array}{c}\text { Delta-Y } \\
\text { inch }\end{array}$ & $\begin{array}{c}\text { Theta-Z } \\
\text { radians }\end{array}$ \\
\hline 1 & $0.00 \mathrm{e}+0$ & $0.00 \mathrm{e}+0$ & $1.35 \mathrm{e}-1$ & $-4.82 \mathrm{e}-3$ \\
2 & $2.17 \mathrm{e}+1$ & $0.00 \mathrm{e}+0$ & $0.00 \mathrm{e}+0$ & $-1.03 \mathrm{e}-2$ \\
3 & $8.53 \mathrm{e}+1$ & $0.00 \mathrm{e}+0$ & $0.00 \mathrm{e}+0$ & $1.03 \mathrm{e}-2$ \\
4 & $1.07 \mathrm{e}+2$ & $0.00 \mathrm{e}+0$ & $1.35 \mathrm{e}-1$ & $4.82 \mathrm{e}-3$ \\
& &
\end{tabular}

Support reactions: (pos. force is to right or up; pos. moment is ccw.)

\begin{tabular}{|c|c|c|c|c|}
\hline $\begin{array}{l}\text { Node } \\
\text { No. }\end{array}$ & $\begin{array}{r}\text { Location } \\
\text { inch }\end{array}$ & $\begin{array}{l}\text { Fx } \\
1 b\end{array}$ & $\begin{array}{l}\text { Fy } \\
\text { Ib }\end{array}$ & $\begin{array}{r}M z \\
1 b-i n\end{array}$ \\
\hline $\begin{array}{l}2 \\
3\end{array}$ & $\begin{array}{l}2.17 e+1 \\
8.53 e+1\end{array}$ & $0.00 \mathrm{e}+0$ & $\begin{array}{l}8.02 e+2 \\
8.03 e+2\end{array}$ & --- \\
\hline
\end{tabular}

Points of internal force discontinuity:

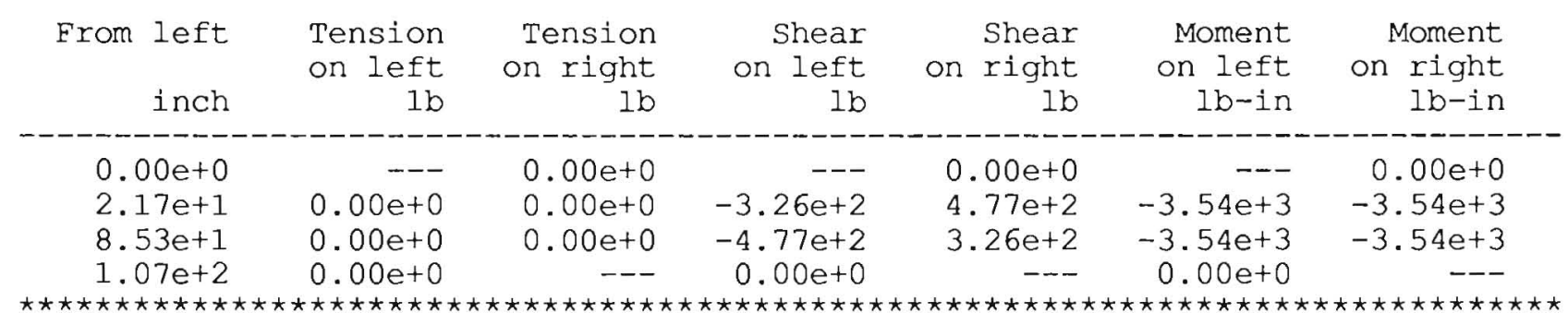

Deformation: (negative deflection is downwards; negative rotation is clockwise)

\begin{tabular}{ccc}
$\begin{array}{c}\text { Location } \\
\text { inch }\end{array}$ & $\begin{array}{c}\text { Rotation } \\
\text { radians }\end{array}$ & $\begin{array}{c}\text { Deflection } \\
\text { inch }\end{array}$ \\
\hline $2.17 \mathrm{e}+1$ & $-1.03 \mathrm{e}-2$ & $0.00 \mathrm{e}+0$ \\
$8.53 \mathrm{e}+1$ & $1.03 \mathrm{e}-2$ & $0.00 \mathrm{e}+0$ \\
& & \\
$0.00 \mathrm{e}+0$ & $-4.82 \mathrm{e}-3$ & $1.35 \mathrm{e}-1$ \\
$1.07 \mathrm{e}+1$ & $-5.48 \mathrm{e}-3$ & $8.12 \mathrm{e}-2$ \\
$2.14 \mathrm{e}+1$ & $-1.01 \mathrm{e}-2$ & $3.25 \mathrm{e}-3$ \\
$3.21 \mathrm{e}+1$ & $-1.33 \mathrm{e}-2$ & $-1.30 \mathrm{e}-1$
\end{tabular}




$\begin{array}{lrr}4.28 e+1 & -8.61 e-3 & -2.53 e-1 \\ 5.35 e+1 & 0.00 e+0 & -3.01 e-1 \\ 6.42 e+1 & 8.61 e-3 & -2.53 e-1 \\ 7.49 e+1 & 1.33 e-2 & -1.30 e-1 \\ 8.56 e+1 & 1.01 e-2 & 3.25 e-3 \\ 9.63 e+1 & 5.48 e-3 & 8.12 e-2 \\ 1.07 e+2 & 4.82 e-3 & 1.35 e-1\end{array}$

$1.07 e+2$

$4.82 e-3$

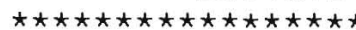
1. $35 e-1$ 
TABLE NO. 23-A—UNIFORM AND CONCENTRATED LOADS

\begin{tabular}{|c|c|c|c|}
\hline \multicolumn{2}{|c|}{ USE OR OCCUPANCY } & \multirow{2}{*}{$\begin{array}{l}\text { UNIFORM } \\
\text { LOAD1 }\end{array}$} & \multirow{2}{*}{$\begin{array}{l}\text { CONCEN- } \\
\text { TRATED } \\
\text { LOAD }\end{array}$} \\
\hline CATEGORY & DESCRIPTION & & \\
\hline \multirow[t]{2}{*}{ 1. Access floor systems } & Office use & 50 & $2000^{2}$ \\
\hline & Computer use & 100 & $2000^{2}$ \\
\hline 2. Armories & & 150 & 0 \\
\hline \multirow{3}{*}{$\begin{array}{l}\text { 3. Assembly areas }{ }^{3} \text { and } \\
\text { auditoriums and } \\
\text { balconies therewith }\end{array}$} & Fixed seating areas & 50 & 0 \\
\hline & $\begin{array}{l}\text { Movable seating and } \\
\text { other areas }\end{array}$ & 100 & 0 \\
\hline & $\begin{array}{l}\text { Stage areas and } \\
\text { enclosed platforms }\end{array}$ & 125 & 0 \\
\hline $\begin{array}{l}\text { 4. Cornices, marquees and } \\
\text { residential balconies }\end{array}$ & & 60 & 0 \\
\hline 5. Exit facilities ${ }^{4}$ & & 100 & $0^{5}$ \\
\hline \multirow[t]{2}{*}{ 6. Garages } & General storage and/or repair & 100 & 6 \\
\hline & $\begin{array}{r}\text { Private or pleasure-type } \\
\text { motor vehicle storage }\end{array}$ & 50 & 6 \\
\hline 7. Hospitals & Wards and rooms & 40 & $1000^{2}$ \\
\hline \multirow[t]{2}{*}{ 8. Libraries } & Reading rooms & 60 & $1000^{2}$ \\
\hline & Stack rooms & 125 & $1500^{2}$ \\
\hline \multirow[t]{2}{*}{ 9. Manufacturing } & Light & 75 & $2000^{2}$ \\
\hline & Heavy & 125 & $3000^{2}$ \\
\hline 10. Offices & & 50 & $2000^{2}$ \\
\hline \multirow[t]{2}{*}{ 11. Printing plants } & Press rooms & 150 & $2500^{2}$ \\
\hline & Composing and linotype rooms & 100 & $2000^{2}$ \\
\hline 12. Residential? & & 40 & $0^{5}$ \\
\hline \multicolumn{4}{|l|}{ 13. Rest rooms ${ }^{8}$} \\
\hline $\begin{array}{l}\text { 14. Reviewing stands, } \\
\text { grandstands and } \\
\text { bleachers }\end{array}$ & & 100 & 0 \\
\hline 15. Roof deck & $\begin{array}{l}\text { Same as area served or for the } \\
\text { type of occupancy } \\
\text { accommodated }\end{array}$ & & \\
\hline 16. Schools & Classrooms & 40 & $1000^{2}$ \\
\hline $\begin{array}{l}\text { 17. Sidewalks and } \\
\text { driveways }\end{array}$ & Public access & 250 & 6 \\
\hline \multirow[t]{2}{*}{ 18. Storage } & Light & 125 & \\
\hline & Heavy & 250 & \\
\hline \multirow[t]{2}{*}{ 19. Stores } & Retail & 75 & $2000^{2}$ \\
\hline & Wholesale & 100 & $3000^{2}$ \\
\hline
\end{tabular}


'See Section 2306 for live load reductions.

2 See Section 2304 (c), first paragraph, for area of load application.

${ }^{3}$ Assembly areas include such occupancies as dance halls, drill rooms, gymnasiums, playgrounds, plazas, terraces and similar occupancies which are generally accessible to the public.

4 Exit facilities shall include such uses as corridors serving an occupant load of 10 or more persons, exterior exit balconies, stairways, fire escapes and similar uses.

Individual stair treads shall be designed to support a 300-pound concentrated load placed in a position which would cause maximum stress. Stair stringers may be designed for the uniform load set forth in the table.

${ }^{6}$ See Section 2304(c), second paragraph, for concentrated loads.

?Residential occupancies include private dwellings, apartments and hotel guest rooms.

8 Rest room loads shall be not less than the load for the occupancy with which they are associated, but need not exceed 50 pounds per square foot. 
Table 2

Minimum Uniformly Distributed Live Loads, $\mathrm{L}_{\mathrm{o}}$

\begin{tabular}{|c|c|c|c|}
\hline Occupancy or Use & $\begin{array}{l}\text { Live Load } \\
\left(\mathrm{lbf} / \mathrm{ft}^{2}\right)\end{array}$ & Occupancy or Use & $\begin{array}{l}\text { Live Load } \\
\left(\mathrm{lb} / / \mathrm{ft}^{2}\right)\end{array}$ \\
\hline Apartments (see residential) & & Manufacturing: & \\
\hline Armories and drill rooms & 150 & Light & 125 \\
\hline \multirow{6}{*}{$\begin{array}{l}\text { Assembly areas and theaters: } \\
\text { Fixed seats (fastened to floor) } \\
\text { Lobbies } \\
\text { Movable seats } \\
\text { Platforms (assembly) } \\
\text { Stage floors }\end{array}$} & & Heavy & 250 \\
\hline & 60 & Marquees and canopies & 75 \\
\hline & 100 & Office buildings: & \\
\hline & 100 & File and computer rooms shall & \\
\hline & 100 & be designed for heavier loads & \\
\hline & 150 & based on anticipated occupancy & \\
\hline \multirow{3}{*}{$\begin{array}{l}\text { Balconies (exterior) } \\
\text { On one- and two-family residences } \\
\text { only, and not exceeding } 100 \mathrm{ft}^{2}\end{array}$} & 100 & Lobbies & 100 \\
\hline & & Offices & 50 \\
\hline & 60 & Penal Institutions: & \\
\hline \multirow{2}{*}{$\begin{array}{l}\text { Bowling alleys, poolrooms, and } \\
\text { similar recreational areas }\end{array}$} & & Cell blocks & 40 \\
\hline & 75 & Corridors & 100 \\
\hline \multirow{4}{*}{$\begin{array}{l}\text { Corridors: } \\
\text { First floor } \\
\text { Other floors, same as occupancy } \\
\text { served except as indicated }\end{array}$} & & Residential: & \\
\hline & 100 & Dwellings (one- and two-family) & \\
\hline & & $\begin{array}{l}\text { Uninhabitable attics } \\
\text { without storage }\end{array}$ & 10 \\
\hline & 100 & Uninhabitable attics & \\
\hline \multirow{3}{*}{$\begin{array}{l}\text { Decks (patio and roof) } \\
\text { Same as area served, or for the } \\
\text { type of occupancy accommodated }\end{array}$} & & $\begin{array}{l}\text { with storage } \\
\text { Habitable attics and sleeping }\end{array}$ & 20 \\
\hline & & $\begin{array}{l}\text { Habitable attics and sleeping } \\
\text { areas }\end{array}$ & \\
\hline & & $\begin{array}{l}\text { areas } \\
\text { All other areas }\end{array}$ & $\begin{array}{l}30 \\
40\end{array}$ \\
\hline Dining rooms and restaurants & 100 & Hotels and multifamily houses & \\
\hline Dwellings (see residential) & & $\begin{array}{l}\text { Private rooms and corridors } \\
\text { serving them }\end{array}$ & 40 \\
\hline \multirow{2}{*}{$\begin{array}{l}\text { Fire escapes } \\
\text { On single-family dwellings only }\end{array}$} & 100 & Public rooms and corridors & \\
\hline & 40 & serving them & 100 \\
\hline \multirow{4}{*}{$\begin{array}{l}\text { Garages (passenger cars only) } \\
\text { For trucks and buses use AASHTO* } \\
\text { lane loads (see Table } 3 \text { for con- } \\
\text { centrated load requirements) }\end{array}$} & 50 & Schools: & \\
\hline & & Classrooms & 40 \\
\hline & & Corridors above first floor & $\ldots 80$ \\
\hline & & Sidewalks, vehicular driveways, & \\
\hline \multirow{2}{*}{$\begin{array}{l}\text { Grandstands (see stadium and arena } \\
\text { bleachers) }\end{array}$} & & and yards, subject to trucking $\dagger$ & 250 \\
\hline & & Stadium and arena bleachers $\ddagger$ & 100 \\
\hline \multirow{2}{*}{$\begin{array}{l}\text { Gymnasiums, main floors and } \\
\text { balconies }\end{array}$} & 100 & Stairs and exitways & 100 \\
\hline & 100 & Storage warehouses: & \\
\hline \multirow{4}{*}{$\begin{array}{l}\text { Hospitals: } \\
\text { Operating rooms, laboratories } \\
\text { Private rooms } \\
\text { Wards } \\
\text { Corridors above first floor }\end{array}$} & & Light & 125 \\
\hline & $\begin{array}{l}60 \\
40\end{array}$ & Heavy & 250 \\
\hline & 40 & Stores: & \\
\hline & 80 & Retail & \\
\hline Hotels (see residential) & & First floor & 100 \\
\hline \multirow{4}{*}{$\begin{array}{l}\text { Libraries: } \\
\text { Reading rooms } \\
\text { Stack rooms - not less than } \S \\
\text { Corridors above first floor }\end{array}$} & & $\begin{array}{l}\text { Upper floors } \\
\text { Wholesale, all floors }\end{array}$ & $\begin{array}{r}75 \\
125\end{array}$ \\
\hline & 60 & $\begin{array}{l}\text { Wholesale, all floors } \\
\text { Walkways and elevated piatforms }\end{array}$ & 125 \\
\hline & 450 & $\begin{array}{l}\text { Walkways and elevated platforms } \\
\text { (other than exitways) }\end{array}$ & हु? \\
\hline & 80 & Yards and terraces (pedestrians) & 100 \\
\hline
\end{tabular}

*American Association of State Highway and Transportation Officials.

†AASHTO lane loads should also be considered where appropriate.

$\ddagger$ For detailed recommendations, see American National Standard for Assembly Seating, Tents, and Air-Supported Structures, ANSI/ NFPA 102-1978.

$\S$ The weight of books and shelving shall be computed using an assumed density of $65 \mathrm{lb} / \mathrm{ft}^{3}$ (pounds per cubic foot, sometimes abbreviated $\mathrm{pcf}$ ) and converted to a uniformly distributed load; this load shall be used if it exceeds $150 \mathrm{lbf} / \mathrm{ft}^{2}$. 


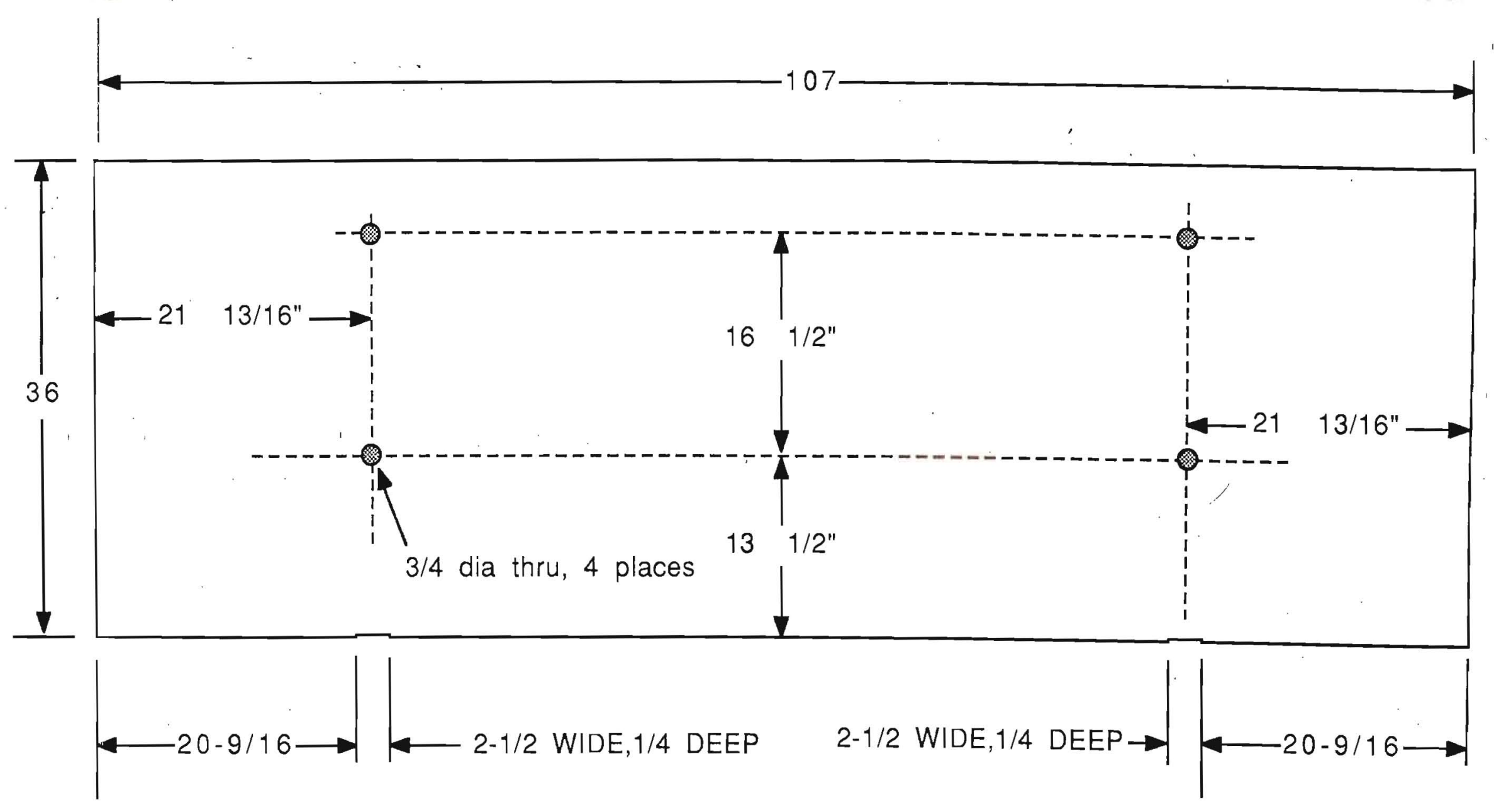

1/8 alum sheet 


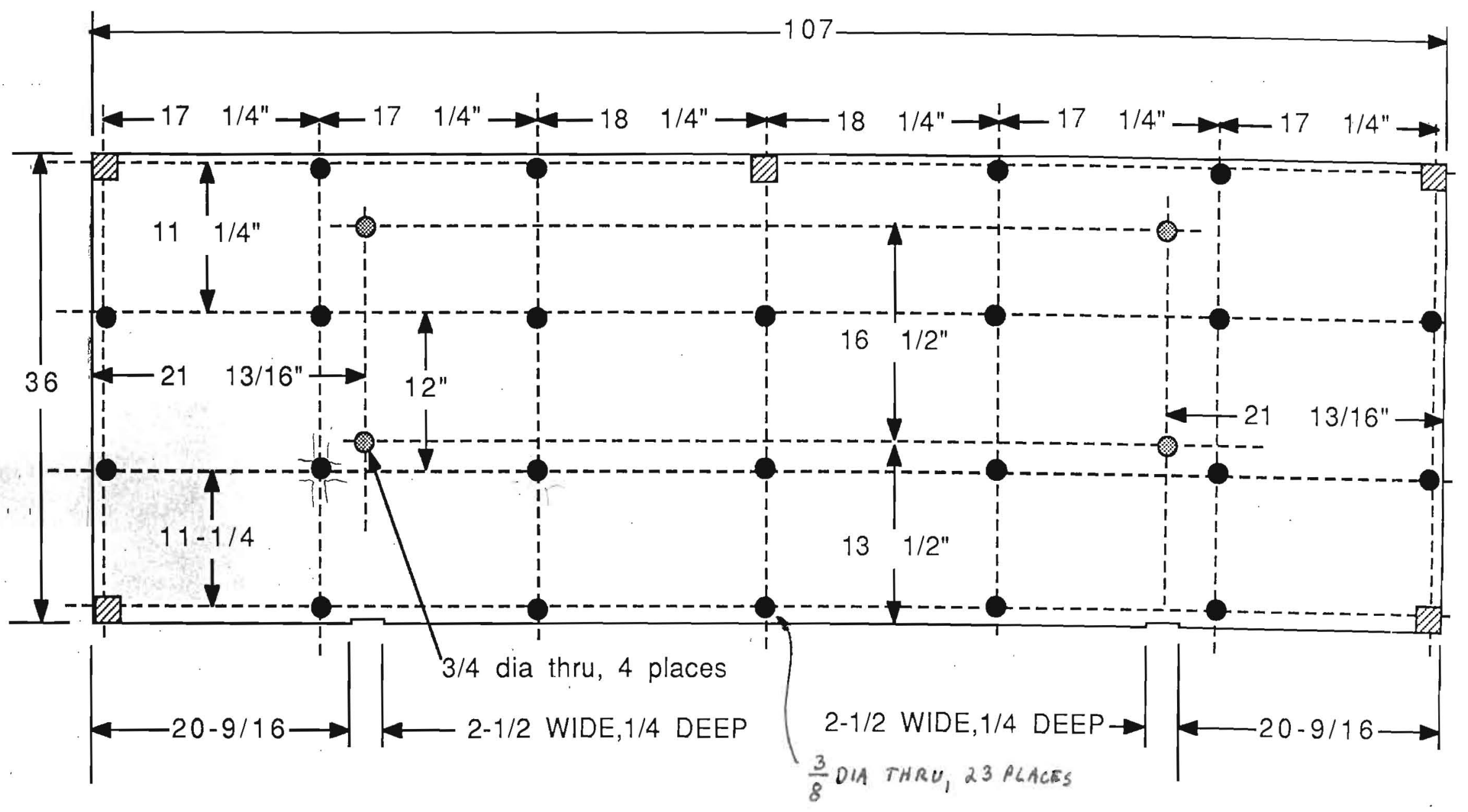

1/16 alum sheet 


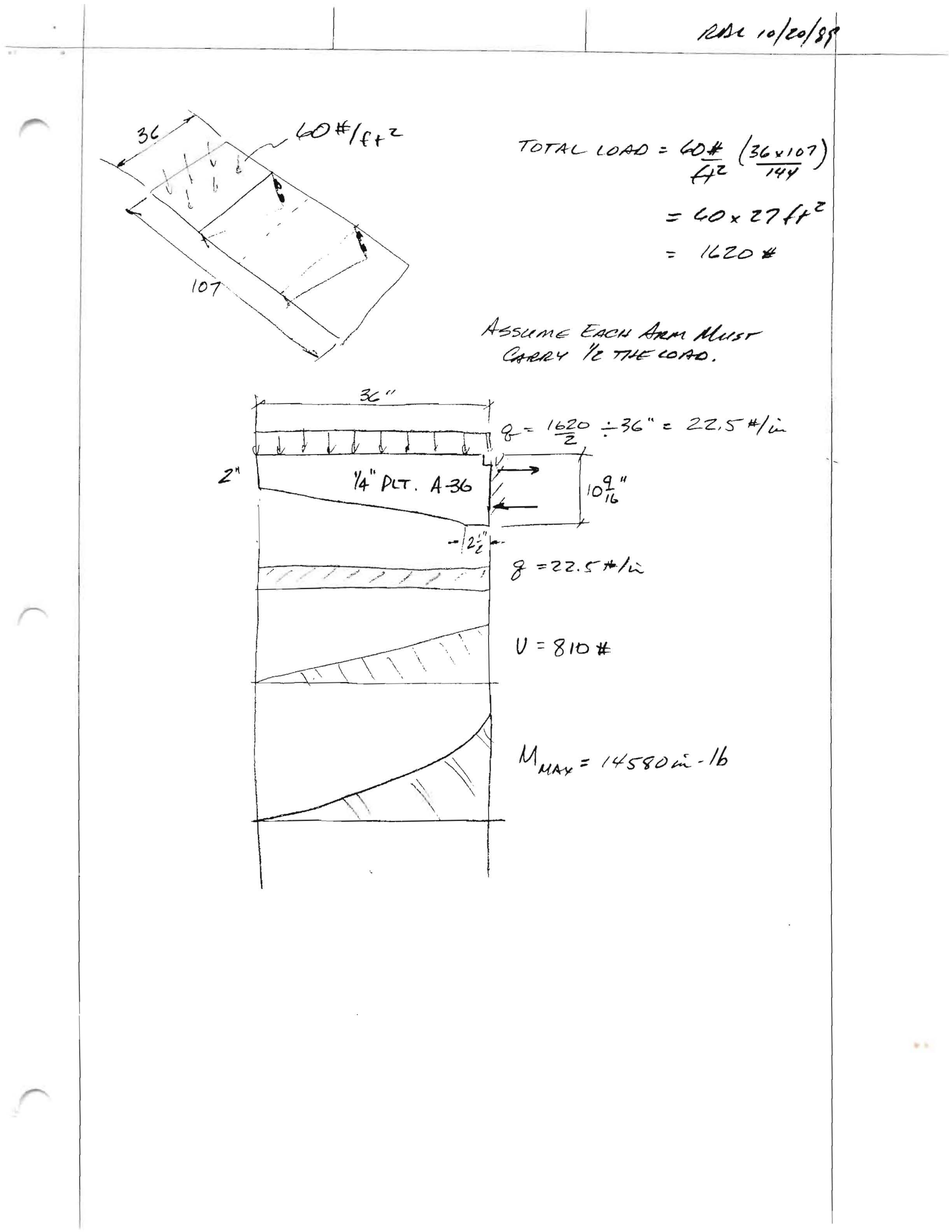


$20010 / 20 / 89$

Careck Pcate

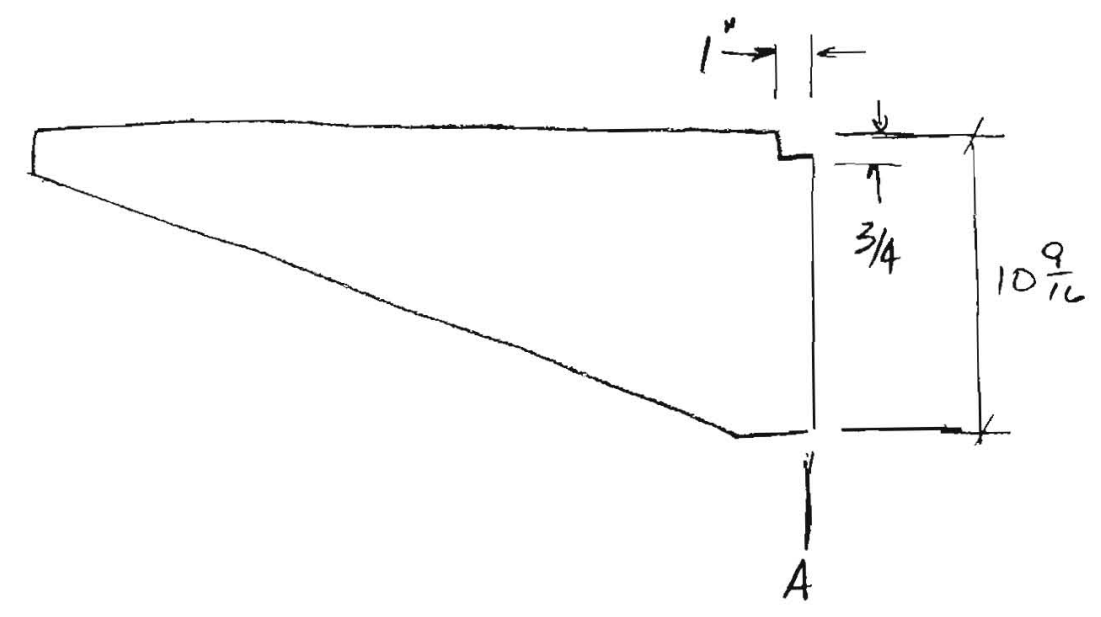

QA

$$
\begin{aligned}
& M_{\text {MAx }}=14580 \text { in } \cdot 16 \\
& \begin{aligned}
-1 / 4 \times 9 \frac{13}{16} \quad I & =\frac{1}{12}\left(\frac{1}{4}\right)\left(\frac{913}{16}\right)^{3}=19,7 \mathrm{in} 4 \\
S & =I=4.01 .3
\end{aligned} \\
& S=\frac{I}{\left(9 \frac{13}{14} / 2\right)}=4.01 \mathrm{in}^{3} \\
& f_{b}=\frac{M}{s}=\frac{14580}{4.01}=3.6 \mathrm{ks} \\
& F_{\text {Tenseor }}=0.6 F_{y}=0.6(36)=21.6 \text { or }
\end{aligned}
$$

Compression: (AISC - 1.5.1.45)

Compute $r_{r} \times \operatorname{lo}^{-1} \frac{4.9 \%}{3}=1.64^{\prime \prime}$

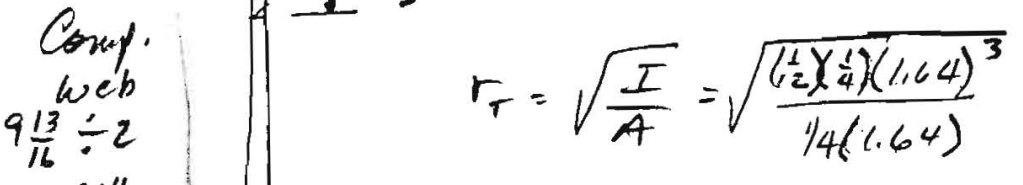

$$
\begin{aligned}
& =0,47^{\prime \prime} \\
& \frac{l}{r_{1}}=\frac{36}{.47}=16.25
\end{aligned}
$$


rese roleolss

$$
\begin{aligned}
& \sqrt{\frac{510 \times 10^{3} C_{b}}{F_{y}}}=\sqrt{\frac{510 \times 10^{3}(1)}{36}}=119 \\
& \sqrt{\frac{102 \times 10^{3} C_{b}}{F_{y}}}=53.2 .3 \\
& F_{b_{c}}=\left[\frac{2}{3}-\frac{F_{y}\left(e / r_{r}\right)^{2}}{1530 \times 10^{3} C_{6}}\right] F_{7}=\left[\frac{2}{3}-\frac{36(76.25)^{2}}{1530 \times 10^{3}(1)}\right] F_{7} \\
& =0.53 F_{4}=.53(36) \\
& F_{b_{\text {comps }}}=19.1 \mathrm{ks},>3.6 \text { OKC F. }>5
\end{aligned}
$$

Chteck Eno Loss of 300

$$
M=300(36)=10800 \mathrm{~s}-16
$$

dess than desigin. OK

Check Itinge.

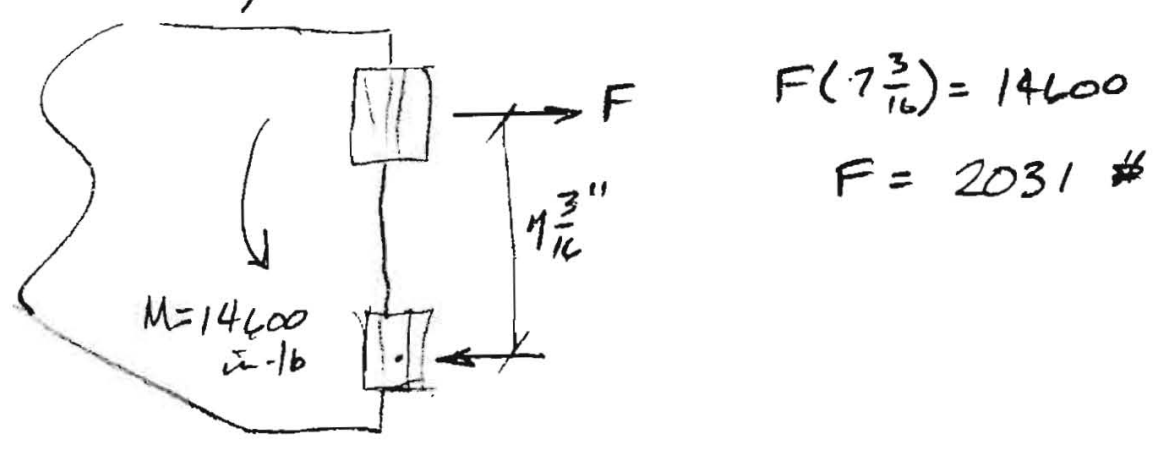


enc $10 / 20 / 59$

Shack 1/4" weld.

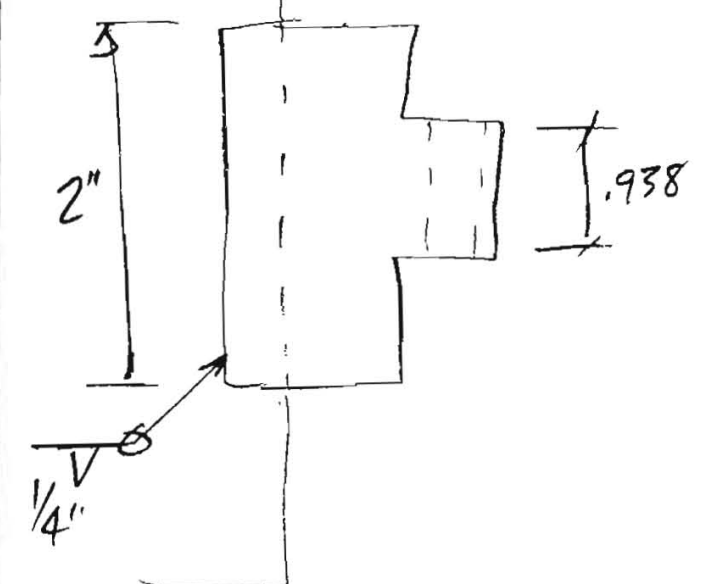

$$
\begin{aligned}
& \text { lempth- } 2 \times 2^{\prime \prime}=4^{\prime \prime}
\end{aligned}
$$

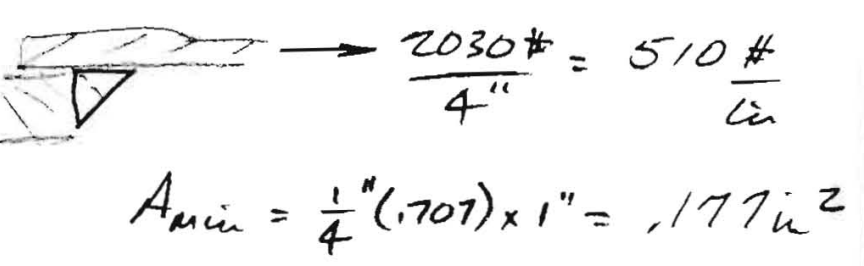

$$
\begin{aligned}
& f_{\mathrm{s}}=\frac{P}{A}=\frac{510}{.177}=2.9 \mathrm{ks} 1 \\
& F_{s}=0,4 F_{y}=0.4(3 c)=14.4 \mathrm{ks} 1 \mathrm{dk} \\
& \text { F.5. } 25
\end{aligned}
$$

Ateckstean@hole

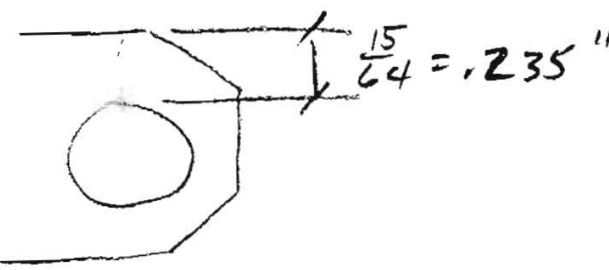

$$
\begin{aligned}
& f_{s}=\frac{P}{A}=\frac{2030}{2(.938)(.235)}=\frac{2030}{._{42}^{2}} \\
& f_{s}=4.6 \mathrm{ks} 1<14.4 \mathrm{ok}
\end{aligned}
$$

the otter $1 / 2$ of the hinge has the same areas

Pin Shear

$\Rightarrow=$

$$
f_{s}=\frac{2030}{\frac{2 \pi}{4}\left(\frac{1}{2}\right)^{2}}=5.2 \mathrm{ks},<14.4010
$$

$d=1 / 2^{n}$

$\therefore$ The supports have. a large margin of Softly. 